\title{
Energy-Efficient Constellations Design and Fast Decoding for Space-Collaborative MIMO Visible Light Communications
}

\author{
Yi-Jun Zhu, Wang-Feng Liang, Chao Wang, and Wen-Ya Wang
}

\begin{abstract}
In this paper, space-collaborative constellations (SCCs) for indoor multiple-input multiple-output (MIMO) visible light communication (VLC) systems are considered. Compared with traditional VLC MIMO techniques, such as repetition coding (RC), spatial modulation (SM) and spatial multiplexing (SMP), SCC achieves the minimum average optical power for a fixed minimum Euclidean distance. We have presented a unified SCC structure for $2 \times 2$ MIMO VLC systems, and extended it to larger MIMO VLC systems with more transceivers. Specifically for $2 \times 2$ MIMO VLC, a fast decoding algorithm is developed with decoding complexity almost linear in terms of the square root of the cardinality of SCC, and the expressions of symbol error rate of SCC are presented. In addition, bit mappings similar to Gray mapping are proposed for SCC. Computer simulations are performed to verify the fast decoding algorithm and the performance of SCC, and the results demonstrate that the performance of SCC is better than those of RC, SM and SMP for indoor channels in general.
\end{abstract}

\section{Index Terms}

Visible light communication (VLC), multiple-input multiple-output (MIMO), space-collaborative constellation, maximum likelihood decoding (ML), bit mapping.

This work was supported in part by Grant No. 61271253 from China National Science Foundation Council and in part by Grant No. 2013AA013603 from China National "863" Program.

Yi-Jun Zhu, Wang-Feng Liang, Chao Wang and Wen-Ya Wang are with National Digital Switching System Engineering \& Technological Research Center, Zhengzhou, Henan Province (450000), China. Emails: yijunzhu1976@outlook.com, 787666734@qq.com,xxgcwangchao@163.com,wwya0202@163.com. 


\section{INTRODUCTION}

In recent decades, due to lower cost, higher power efficiency and longer lifetime, the replacement of traditional lighting equipments with light-emitting diodes (LEDs) has led to the development of visible light communications (VLCs), which is proposed as an appealing way to provide high speed network access [1]-[3].

In a practical indoor environment, multiple lamps are simultaneously utilized to meet the illumination requirement, therefore, the combination of VLC and multiple-input multiple-output (MIMO) technique is a nature method to provide high data-rate transmission [4], [5]. However, the channel in an indoor VLC system is typically deterministic and the performance of MIMO VLC systems relies heavily on the channel correlations [6], [7].

Among those MIMO techniques utilized in indoor VLC systems, repetition coding (RC) is the simplest one. RC emits the same signal from all the transmitters simultaneously and achieves good performance for highly correlated channels [8]. However, when the channel matrix is diagonal, RC does not provide any channel multiplexing and thus performs inefficiently. On the contrary, spatial multiplexing (SMP) transmits different signals from different LEDs, bringing the highest spectrum efficiency when channel correlations are sufficiently low [9], [10]. Furthermore, spatial modulation (SM) is also an MIMO VLC technique which actives only one of the LEDs to transmit signal at any time instance in order to avoid inter-channel interference (ICI) [11], [12].

On the other side, indoor VLC has some unique advantages, such as abundant space resource, channel static stability, physically controlled coverage and so on. Recently, many techniques, such as fisheye lens [13] and angle diversity receivers [14], have been introduced to construct a physically controlled channel to realize better performance for MIMO VLC systems. In order to deal with interference for a VLC system with two LEDs and two users, an optimization problem is formulated to decide the mapping from data bits to LED outputs such that the bit error rates (BERs) of both users are reduced [15]. A novel constellation named as space-collaborative constellation (SCC) for $2 \times 2$ MIMO VLC was designed in [6], where the fundamental conception of SCC was presented and traditional maximum likelihood (ML) detection relied on exhaustive search was used. Compared with traditional VLC MIMO techniques, such as RC, SM and SMP, SCC achieved the minimum average optical power for a fixed minimum Euclidean distance. 
In this paper, Furthermore, we will develop a fast decoding algorithm, whose decoding complexity is almost linear in terms of the square root of the cardinality of SCC. In addition, we will propose bit mappings for SCC. Computer simulations will verify the fast decoding algorithm and show the average performance of SCC under mobile channel environment where the receiver is moving randomly within the room.

The main contributions of this paper can be summarized as follows:

- We will present a unified structure of SCC in [6] for $2 \times 2$ MIMO VLC systems, and extend it to larger MIMO VLC systems with more transceivers.

- Specifically for $2 \times 2$ MIMO VLC, a fast decoding algorithm called zero-forcing detection and minimum distance decoding (ZF-MD) will be developed, whose decoding complexity is almost linear in terms of the square root of the cardinality of SCC. When the channel matrix is an identity matrix, we will prove that the performance of ZF-MD detection is the same as that of joint maximum likelihood (JML) detection.

- Specifically for $2 \times 2$ MIMO VLC, the expressions of symbol error rate (SER) of SCC are presented.

- We will propose bit mappings for two-dimensional SCC. Computer simulations will show the average bit error performance of SCC under mobile channel environment where the receiver is moving randomly within the room.

Notation: Most notations used throughout this paper are standard: Boldface upper-case letters denote matrices, boldface lower-case letters denote column vectors, and standard lower-case letters denote scalars. Notation $\|\mathbf{r}\|_{m}$ denotes a $m$-norm of $\mathbf{r}$. $\mathbb{E}[\cdot]$ denotes the expected value of the expression in brackets. $\lfloor\cdot\rfloor$ is the floor function; $\lceil\cdot\rceil$ is the ceiling function; $\bmod (a, b)$ denotes modulus $b$ operation on $a$, i.e., $\bmod (5,4)=1, \bmod (-2,4)=2$.

\section{Collaborative Constellations}

We consider an indoor visible light communication system with $M$ transmitting antennas (LEDs) and $N$ receiving antennas (photo-detectors). Its discrete-time baseband MIMO channel model can be represented as

$$
\mathbf{r}=\mathbf{H} \mathbf{s}+\mathbf{n},
$$

where $\mathbf{n}$ is the sum of ambient shot light noise and thermal noise. It is independent of the transmitted signals [1], [9], [10]. Consequently, $\mathbf{n}$ is real valued additive white Gaussian noise 
(AWGN) with zero mean and a variance $\sigma^{2}=\sigma_{\text {shot }}^{2}+\sigma_{\text {thermal }}^{2}$, where $\sigma_{\text {shot }}^{2}$ is the shot noise variance and $\sigma_{\text {thermal }}^{2}$ is the thermal noise variance [1], [9], [10]. It is assumed that $M$ data symbols $s_{1}, s_{2}, \cdots, s_{M}$ are chosen randomly, equally-likely and independently to form an input data vector $\mathbf{s}=\left[s_{1}, s_{2}, \cdots, s_{M}\right]^{T} \in \mathcal{X}^{M}$, where $\mathcal{X}$ is a given modulation constellation. The received signal vector is denoted by $\mathbf{r}=\left[r_{1}, r_{2}, \cdots, r_{N}\right]^{T}$. Notation $\mathbf{H}$ denotes an $N \times M$ channel matrix, in which $h_{n m}$ is the non-negative and real coefficient between the $m$-th transmitter antenna and the $n$-th receiver antenna. In this paper, we assume an indoor environment with light-of-sight (LOS) characteristic, where the channel gain of a VLC link is determined by [1], [5]

$$
h_{n m}=\left\{\begin{array}{cc}
\frac{(k+1) A}{2 \pi d_{n m}^{2}} \cos ^{k}(\phi) \cos (\psi), 0 \leq \psi \leq \Psi_{1 / 2} \\
0, & \psi>\Psi_{1 / 2}
\end{array},\right.
$$

with $\phi$ being the angle of emergence with respect to the $m$-th transmitter axis and $\psi$ being the angle of incidence with respect to the $n$-th receiver axis. In addition, $d_{n m}$ is the distance between $m$-th transmitter and $n$-th receiver. $\Psi_{1 / 2}$ is the field-of-view (FOV) angle of the receiver, $A$ is the detector area of the receiver, and $k=\frac{-\ln 2}{\ln \left(\cos \left(\Phi_{\frac{1}{2}}\right)\right)}$, in which $\Phi_{1 / 2}$ is the half power angle of the transmitter.

In VLC systems, the average optical power $P_{a o}$ is important to meet lighting requirement and eye-safety limitations, which is defined as $P_{a o}=\frac{c \mathbb{E}\left[\|\mathbf{s}\|_{1}\right]}{\sqrt{T_{s}}}$, where $c$ represents the electro-optical conversion factor in watts per ampere (W/A), and $T_{s}$ is the symbol period [16]-[18]. We let $\frac{c}{\sqrt{T_{s}}}=1$ for simplicity here. Thus, if we use notation $P(\Omega)$ to denote the average optical power of constellation $\boldsymbol{\Omega}$, then $P(\boldsymbol{\Omega})$ can be expressed by $P(\boldsymbol{\Omega})=\frac{1}{|\boldsymbol{\Omega}|} \sum_{\mathbf{s} \in \boldsymbol{\Omega}}\|\mathbf{s}\|_{1}$, where notation $|\boldsymbol{\Omega}|$ denotes the cardinality of $\Omega$.

The primary goal of designing a collaborative constellation $\Omega, \mathrm{s} \in \Omega$, is to achieve optimal power-efficient subject to a fixed minimum Euclidean distance. Hence, the corresponding optimization problem can be formally stated as [6]

$$
\begin{array}{ll}
\text { Minmize } & P(\boldsymbol{\Omega})=\mathbb{E}\left[\|\mathbf{s}\|_{1}\right], \\
\text { Subject to } & |\boldsymbol{\Omega}|=L, \\
& d_{\min }(\boldsymbol{\Omega})=1 .
\end{array}
$$

where $d_{\min }(\boldsymbol{\Omega})=\min _{\mathbf{s} \neq \mathbf{s}^{\prime} \in \boldsymbol{\Omega}}\left|\mathbf{s}-\mathbf{s}^{\prime}\right|$ and the symbols $s_{m}, m=1,2, \ldots, M$, in the signal vector $\mathbf{s}$ are all non-negative integers; $L$ is the constellation size. The solution to (3) is given by the following theorem. 
Theorem 1: [6] For a MIMO VLC system with 2 transmitters, let $\mathbf{s}=(x, y)^{T}$ be the transmitted symbol, the optimal collaborative constellation $\Omega$ is determined by $\Omega=\bigcup_{j=0}^{\lambda} \Omega_{j}$,

$$
\boldsymbol{\Omega}_{j}=\left\{(x, y)^{T}: x+y=j, 0 \leq x, y \in \mathbb{Z}\right\}
$$

for $0 \leq j \leq \lambda-1, \lambda=\left\lceil\frac{\sqrt{8 L+1}-3}{2}\right\rceil$, and $\Omega_{\lambda}$ consists of any $\bar{L}=L-\lambda(\lambda+1) / 2$ solutions of $x+y=\lambda, 0 \leq x, y \in \mathbb{Z}$. Furthermore, the optimal average optical power of $\Omega$ is given by $P(\boldsymbol{\Omega})=\lambda-\frac{\lambda^{3}+3 \lambda^{2}+2 \lambda}{6 L}$.

Then, we would like to make the following comments about Theorem 1.

1) The symbols of SCC are generated collaboratively. In traditional MIMO VLC schemes, such as RC, SMP and SM, the transmitted symbols through different LEDs are usually generated independently. The symbols of RC are generated from the diagonal line and those of SM are generated from the two coordinate axes. However, the symbols $x, y$ are selected jointly to satisfy $x+y=j, 0 \leq j \leq \lambda$, in the new constellation. Hence, the transmitted symbols through LEDs located different space are generated collaboratively, so the new constellation is called as space collaborative constellations.

2) The symbols of SCC should be detected jointly at the receiver side. For example, let $L=16, H=[2 D, D ; D, 2 D]$, where $D$ is a real positive number. Consider the MIMO channel model in (1) and assume $n=0$. Then for all the following transmitted symbols $(2,0)^{T},(1,2)^{T},(0,4)^{T}$, the received signal $r_{1}$ equals to $4 D$. Also, for all the following transmitted symbols $(0,2)^{T},(2,1)^{T},(4,0)^{T}$, the received signal $r_{2}$ equals to $4 D$. Therefore, if the symbols are detected independently, the decoder can not recover the transmitted symbols correctly with such ambiguity. However, if the symbols are detected jointly, for all the following transmitted symbols $(2,0)^{T},(1,2)^{T},(0,4)^{T}$, the received signal vector $\left(r_{1}, r_{2}\right)^{T}$ equals to $(4 D, 2 D)^{T},(4 D, 5 D)^{T},(4 D, 8 D)^{T}$, respectively. Also, for all the following transmitted symbols $(0,2)^{T},(2,1)^{T},(4,0)^{T}$, the received signal vector $\left(r_{1}, r_{2}\right)^{T}$ equals to $(2 D, 4 D)^{T},(5 D, 4 D)^{T},(8 D, 4 D)^{T}$, respectively. We can observe that the symbols can be recovered uniquely if and only if joint detection is used.

However, we can observe that $\Omega_{j}, j=0,1, \ldots, \lambda-1$, are fixed in Theorem 1 , but $\Omega_{\lambda}$ consists of any $\bar{L}=L-\lambda(\lambda+1) / 2$ solutions of $x+y=\lambda$, so the constellation presented in Theorem 1 is not unique. Furthermore, the average optical power of each LED should be equal, so as to yield consistent illumination level over the room which is important to meet lighting requirement. Here, 
we present a unified structure of the collaborative constellations with some special properties in the following Lemma.

Lemma 1: When $L=2^{2 q+1}, q=1,2,3, \ldots$, one of the collaborative constellation $\Omega$ for MIMO VLC with two transmitters is determined by $\lambda=\sqrt{2 L}-1, \Omega_{j}=\left\{(x, y)^{T}: x+y=j, 0 \leq x, y \in\right.$ $\mathbb{Z}\}$, for $0 \leq j \leq \lambda-1$ and $\Omega_{\lambda}=\left\{(x, y)^{T}: x+y=\lambda, \bmod (x, 2)=0,0 \leq x<\lambda / 2\right\} \cup\left\{(x, y)^{T}:\right.$ $x+y=\lambda, \bmod (y, 2)=0,0 \leq y<\lambda / 2\}$. Moreover, the total optical power of constellation $\Omega$ is optimal, and the average optical power of each LED is equal.

When $L=8,32,128,512$, the space collaborative constellations are shown in Fig. 1(a), where $\mathrm{x}$-axis and $\mathrm{y}$-axis represent the optical power of LED 1 and LED 2, respectively. It can be seen that SCC is shaped like a triangle, and there are just $(\lambda+1) / 2$ symbols in $\Omega_{\lambda}$. Then, we would like to make the following comments about Lemma 1.

1) The average optical power of each LED is equal. We can observe that Fig. 1(a) is symmetry about line $y=x$, so the average optical power of each LED is equal. Furthermore, it can be calculated that the average optical power of each LED is $\frac{\lambda}{2}-\frac{\lambda^{3}+3 \lambda^{2}+2 \lambda}{12 L}$.

2) The error performance of the constellation presented in Lemma 1 is optimal. When $L=8,32,128,512, \ldots$, there are $(\lambda+1) / 2$ points in $\Omega_{\lambda}$, so there are $\left(\begin{array}{c}\lambda+1 \\ (\lambda+1) / 2\end{array}\right)$ possible constellations in Theorem 1 in total. The minimum Euclidian distance of all the possible constellations are equal to 1 , therefore, the error performance difference is determined by the kissing numbers. We can observe that when the point is on the x-axis or y-axis, the kissing number will be added one; but when the point is not on the axes, the kissing number will be added two. For the $\Omega_{\lambda}$ presented in Lemma 1, there are one point on the $\mathrm{x}$-axis and $\mathrm{y}$-axis, respectively. Therefore, its kissing number is the smallest and its error performance is optimal.

When $L=4,16,64$, there is no unified structure for SCC, and the corresponding constellations we proposed are shown in Fig. 1(b). Compared Fig. 1(a) with Fig. 1(b), the only difference is that the points on the diagonal line have no unified form when $L=4,16,64, \ldots$.

Average optical power of each LED at different spectral efficiency for MIMO VLC systems with two transmitters is shown in Tab. I. We can observe that the average optical power of each LED is equal in general. The only exceptional case is when $L=16$, as shown in the second sub-figure of Fig. 1(b). We remove the point $(2,2)$ and add a new point $(3,2)$, then a new 
constellation for $L=16$ is obtained, as shown in the third sub-figure of Fig. 1(b). From Tab. I, we can observe that the second sub-figure is more energy efficient, but the third one achieves equal power for each LED.

Lemma 2: For a MIMO VLC system with $M$ transmitters, let $\mathbf{s}=\left(s_{1}, s_{2}, \ldots, s_{M}\right)^{T}$ be the transmitted symbol vector, the optimal collaborative constellation $\Omega$ is determined by $\Omega=$ $\bigcup_{j=0}^{\lambda} \Omega_{j}$, and

$$
\boldsymbol{\Omega}_{j}=\left\{\mathbf{s}: \sum_{i=1}^{M} s_{i}=j, 0 \leq s_{1}, s_{2}, \ldots, s_{M} \in \mathbb{Z}\right\},
$$

where $\lambda$ is determined by $\sum_{j=0}^{\lambda-1}\left|\Omega_{j}\right|<L \leq \sum_{j=0}^{\lambda}\left|\Omega_{j}\right|$.

TABLE I

AVERAGE OPTICAL POWER OF EACH LED AND THE AVERAGE OPTICAL POWER OF SCC AT DIFFERENT SPECTRAL EFFICIENCY FOR MIMO VLC SYSTEMS WITH TWO TRANSMITTERS

\begin{tabular}{|c||c|c|c|}
\hline Constellation Size & $P_{\mathrm{LED}_{1}}$ & $P_{\mathrm{LED}_{2}}$ & Average Power \\
\hline$L=4$ & 0.5 & 0.5 & 1 \\
\hline$L=8$ & 0.875 & 0.875 & 1.75 \\
\hline$L_{a}=16$ & 1.4375 & 1.375 & 2.8125 \\
\hline$L_{b}=16$ & 1.4375 & 1.4375 & 2.875 \\
\hline$L=32$ & 2.1875 & 2.1875 & 4.375 \\
\hline$L=64$ & 3.28125 & 3.28125 & 6.5625 \\
\hline$L=128$ & 4.84375 & 4.84375 & 9.6875 \\
\hline$L=256$ & 7.046875 & 7.046875 & 14.09375 \\
\hline$L=512$ & 10.171875 & 10.171875 & 20.34375 \\
\hline
\end{tabular}

Based on Lemma 2, collaborative constellation graphs for the MIMO VLC systems with three transmitters when $L=16,32,64,128$ are shown in Fig. 2. It can be seen that the constellation is shaped like a pyramid.

Presently, there are three commonly-used transmission schemes for the MIMO VLC systems, such as RC, SM, and SMP. If the modulation is pulse amplitude modulation (PAM) with an order of $r_{1}$, the spectral efficiency of $\mathrm{RC}$ is $R_{R C}=\log _{2} r_{1}(\mathrm{bit} / \mathrm{s} / \mathrm{Hz})$, and its average optical 


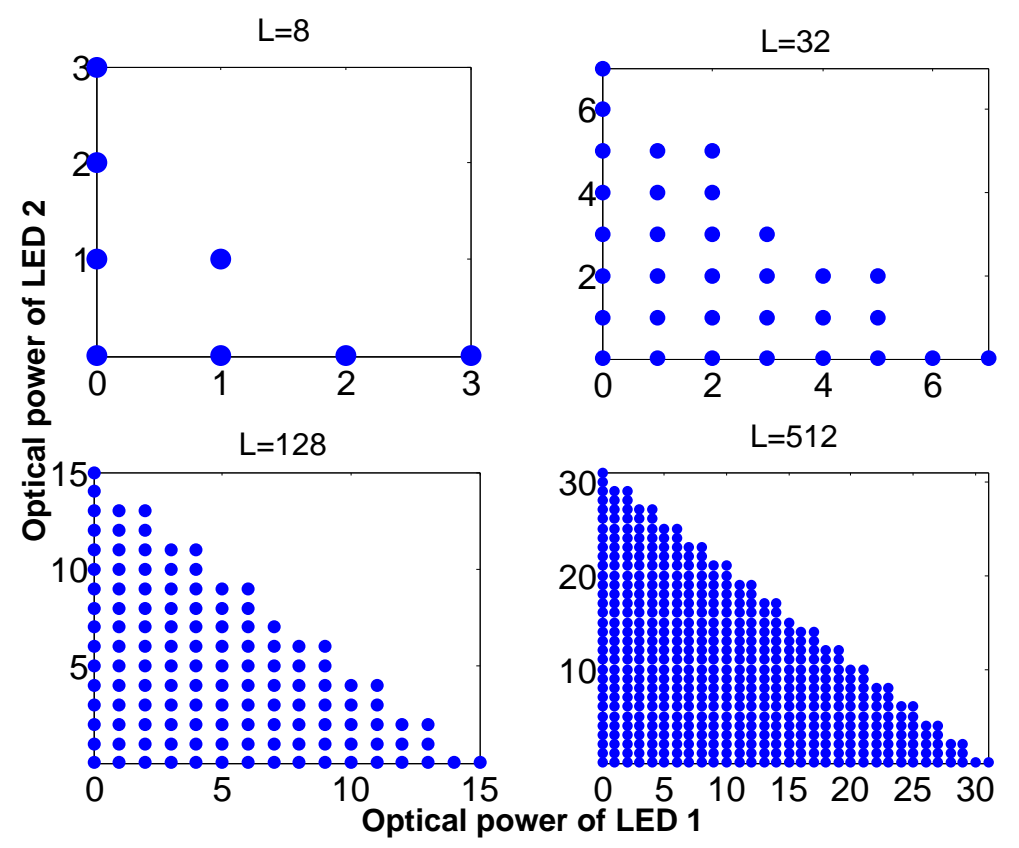

(a) $L=8,32,128,512$

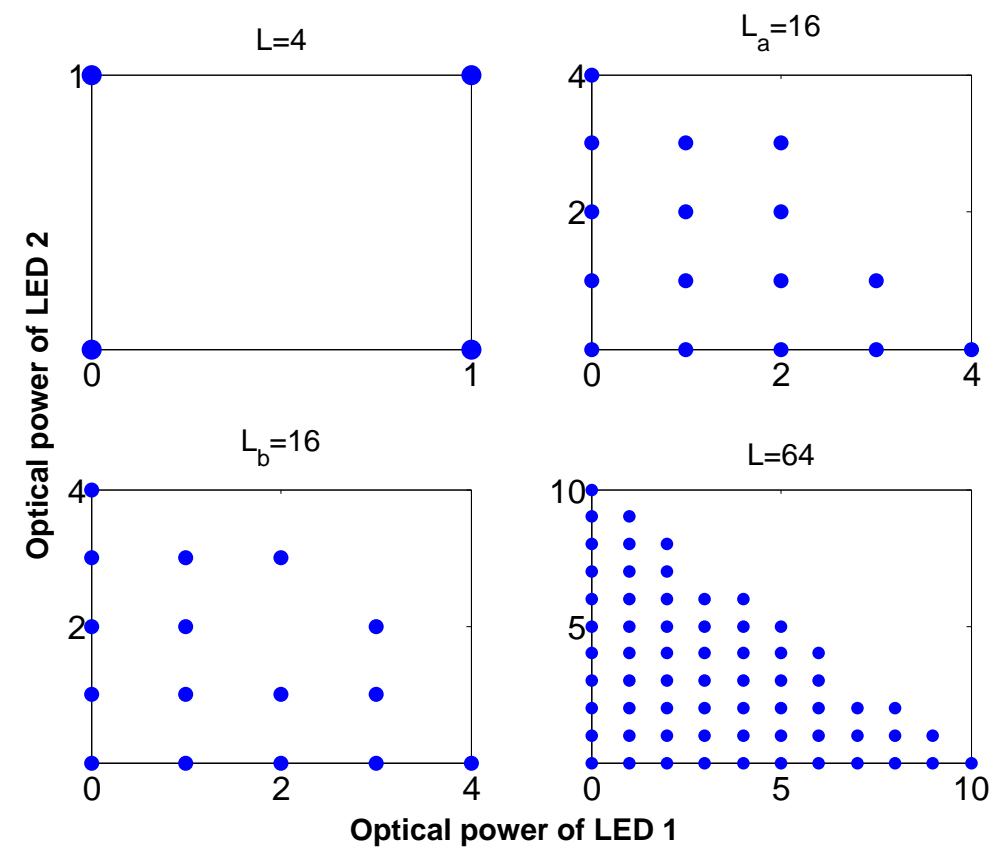

(b) $L=4,16,64,256$

Fig. 1. Constellations of SCC for MIMO VLC systems with two transmitters. 

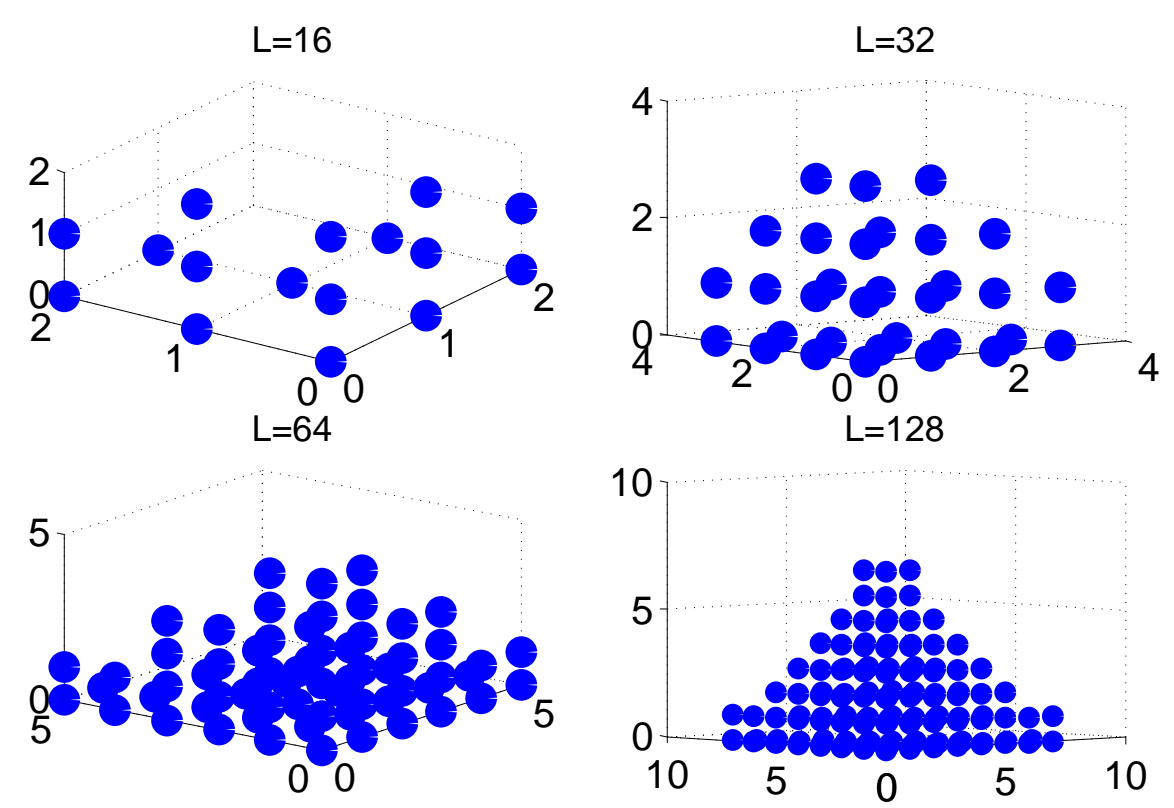

Fig. 2. Constellations of SCC for MIMO VLC systems with three transmitters.

power is $P_{R C}=r_{1}-1$. If the modulation is $r_{2}$-PAM, SM provides a spectral efficiency as $R_{S M}=\log _{2} M+\log _{2} r_{2}(\mathrm{bit} / \mathrm{s} / \mathrm{Hz})$, and its average optical power is $P_{S M}=\frac{r_{2}+1}{2}$. SMP provides a spectral efficiency as $R_{S M P}=M \log _{2} r_{3}(\mathrm{bit} / \mathrm{s} / \mathrm{Hz})$, and its average optical power is $P_{S M P}=$ $r_{3}-1$,where $r_{3}$ is the modulation order. When the constellation size of SCC is $L, R=\log _{2} L$ is the corresponding spectral efficiency. Average normalized optical power for SM, RC, SMP and SCC with different spectral efficiency is shown in Tab. II, we will see that SCC achieves the minimal average optical power. Furthermore, average optical power of each LED at different spectral efficiency for MIMO VLC systems with three transmitters is shown in Tab. III. We can observe that the average optical power of each LED is equal. 
TABLE II

AVERAGE OPTICAL POWER OF SM, RC, SMP AND SCC AT DIFFERENT SPECTRAL EFFICIENCY FOR MIMO VLC SYSTEMS WITH THREE TRANSMITTERS

\begin{tabular}{|c||c|c|c|c|}
\hline Constellation Size & SM & RC & SMP & SCC \\
\hline$L=4$ & 1.25 & 4.5 & 1 & 0.75 \\
\hline$L=8$ & 1.875 & 10.5 & 1.5 & 1.375 \\
\hline$L=16$ & 3.1875 & 22.5 & 2.5 & 2.0625 \\
\hline$L=32$ & 5.8438 & 46.5 & 3.5 & 2.9063 \\
\hline$L=64$ & 11.1719 & 94.5 & 4.5 & 4.0313 \\
\hline$L=128$ & 21.8359 & 190.5 & 6.5 & 5.4219 \\
\hline$L=256$ & 43.168 & 382.5 & 8.5 & 7.207 \\
\hline$L=512$ & 85.834 & 766.5 & 10.5 & 9.4453 \\
\hline
\end{tabular}

TABLE III

AVERAGE OPTICAL POWER OF EACH LED AND THE AVERAGE OPTICAL POWER OF SCC AT DIFFERENT SPECTRAL EFFICIENCY FOR MIMO VLC SYSTEMS WITH THREE TRANSMITTERS

\begin{tabular}{|c||c|c|c|c|}
\hline Constellation Size & $P_{\mathrm{LED}_{1}}$ & $P_{\mathrm{LED}_{2}}$ & $P_{\mathrm{LED}_{3}}$ & Average Power \\
\hline$L=4$ & 0.25 & 0.25 & 0.25 & 0.75 \\
\hline$L=8$ & 0.5 & 0.5 & 0.5 & 1.5 \\
\hline$L=16$ & 0.6875 & 0.6875 & 0.6875 & 2.0625 \\
\hline$L=32$ & 0.96875 & 0.96875 & 0.96875 & 2.90625 \\
\hline$L=64$ & 1.359375 & 1.359375 & 1.359375 & 4.078125 \\
\hline$L=128$ & 1.8125 & 1.8125 & 1.8125 & 5.4375 \\
\hline$L=256$ & 2.40234375 & 2.40234375 & 2.40234375 & 7.20703125 \\
\hline$L=512$ & 3.1484375 & 3.1484375 & 3.1484375 & 9.4453125 \\
\hline
\end{tabular}

For the MIMO VLC systems with four transmitters, it is not so easy to draw the graphs of four-dimensional constellations. Average optical power for SM, RC, SMP and SCC with different spectral efficiency is shown in Tab. IV, we will see that SCC still achieves the minimal average optical power. 
TABLE IV

AVERAGE OPTICAL POWER OF SM, RC, SMP AND SCC AT DIFFERENT

SPECTRAL EFFICIENCY FOR MIMO VLC SYSTEMS WITH FOUR

TRANSMITTERS

\begin{tabular}{|c||c|c|c|c|}
\hline Constellation Size & SM & RC & SMP & SCC \\
\hline$L=4$ & 1 & 6 & 1 & 0.75 \\
\hline$L=8$ & 1.5 & 14 & 1.5 & 1.25 \\
\hline$L=16$ & 2.5 & 30 & 2 & 1.6875 \\
\hline$L=32$ & 4.5 & 62 & 3 & 2.34375 \\
\hline$L=64$ & 8.5 & 126 & 4 & 3.125 \\
\hline$L=128$ & 16.5 & 254 & 5 & 4.03125 \\
\hline$L=256$ & 32.5 & 510 & 6 & 5.19531125 \\
\hline$L=512$ & 64.5 & 1022 & 8 & 6.486328125 \\
\hline
\end{tabular}

\section{Fast Decoding Algorithm And Symbol Error Rate of SCC}

The primary purpose of this section is to develop a fast decoding algorithm and to analysis the symbol error rates for space collaborative constellations. We consider a $2 \times 2$ MIMO VLC system and we assume that the channel state information is completely available at the receiver.

Since the symbols through two LEDs in SCC are generated collaboratively, the optimal receiver to jointly estimate the transmitted symbols of SCC is the JML detector, which is to solve the following optimization problem:

$$
\hat{\mathbf{s}}=\arg \min _{\mathbf{s} \in \mathbf{\Omega}}\|\mathbf{r}-\mathbf{H} \mathbf{s}\|^{2} .
$$

From (6), it can be seen that traditional JML decoding relies on exhaustive comparisons, whose complexity is determined by $\mathcal{O}(|\Omega|)$ and thus, very high for increasing date rate. Therefore, in the following, our main task is to develop a low-complexity receiver, called zero-forcing minimum distance based receiver, which consists of two successive steps: zero-forcing detection and minimum distance decoding. 


\section{A. Zero-Forcing Detector}

According to (1), we can obtain a received model with a zero-forcing (ZF) detector,

$$
\tilde{\mathbf{r}}=\mathbf{s}+\mathbf{H}^{-1} \mathbf{n}
$$

where $\tilde{\mathbf{r}}=\left(r_{x}, r_{y}\right)^{T}$. Then, we can estimate the transmitted symbols of SCC as follows

$$
\hat{\mathbf{s}}=\arg \min _{\mathbf{s} \in \mathbf{\Omega}}\|\tilde{\mathbf{r}}-\mathbf{s}\|^{2} .
$$

Compare (6) with (8), we can observe that if and only if $\mathbf{H}=\mathbf{I}_{2}$, the performance of ZF detector is equal to that of ML detector; otherwise, $\mathrm{ZF}$ detector will lead to some performance loss. These are the same as those in radio frequency $(\mathrm{RF})$ communications.

\section{B. Minimum Distance Decoding}

For $2 \times 2$ MIMO VLC systems using SMP, two independent PAM symbols are transmitted respectively by the transmitter apertures. For this SMP system, after ZF detection, the optimal estimates of the two transmitted PAM symbols can be independently obtained from the corresponding entry of the output vector of ZF detector. However, for our SCC based MIMOOWC, the two entries of the $\mathrm{ZF}$ output are not independent since the transmitted space vector entries are collaboratively designed. Therefore, the decoding of our SCC can not following that of SMP in a straightforward manner. Indeed, we are motivated to develop a fast decoding algorithm to compute (8), which is described as follows.

Theorem 2: Let $\tilde{x}=\operatorname{Quant}\left[r_{x}\right], \tilde{y}=\operatorname{Quant}\left[r_{y}\right], m=\bmod (\lambda-|\tilde{x}-\tilde{y}|, 4), n=\left\lfloor\frac{\lambda-|\tilde{x}-\tilde{y}|}{4}\right\rfloor$, when $L=8,32,128, \ldots$, the minimum distance estimation vector $\hat{\mathbf{s}}=(\hat{x}, \hat{y})^{T}$ of $2 \times 2 \mathrm{MIMO}$ is determined by

1) If $\tilde{x}+\tilde{y}<\lambda, \hat{\mathbf{s}}=(\tilde{x}, \tilde{y})^{T}$;

2) If $\tilde{x}+\tilde{y}=\lambda$, then $\hat{\mathbf{s}}$ is determined by

$$
\hat{\mathbf{s}}= \begin{cases}(\tilde{x}, \tilde{y})^{T}, & \text { if } m=0 \\ (\tilde{x}-1, \tilde{y})^{T}, & \text { else if } m>0 \text { and } r_{x}-r_{y} \leq \tilde{x}-\tilde{y} \\ (\tilde{x}, \tilde{y}-1)^{T}, & \text { else. }\end{cases}
$$


3) If $\tilde{x}+\tilde{y}>\lambda$, then $\hat{\mathbf{s}}$ is determined by

$$
\hat{\mathbf{s}}= \begin{cases}(\lambda-2 n, 2 n)^{T}, & \text { if } m=0 \text { and } \tilde{x}-\tilde{y} \geq 0 ; \\ (2 n, \lambda-2 n)^{T}, & \text { elseif } m=0 \text { and } \tilde{x}-\tilde{y}<0 ; \\ (\lambda-2 n-1,2 n+1)^{T}, & \text { else if } m>0 \text { and } \tilde{x}-\tilde{y} \geq 0 \text { and } r_{x}-r_{y} \leq \tilde{x}-\tilde{y} ; \\ (\lambda-2 n+1,2 n-1)^{T}, & \text { else if } m>0 \text { and } \tilde{x}-\tilde{y} \geq 0 \text { and } r_{x}-r_{y}>\tilde{x}-\tilde{y} ; \\ (2 n-1, \lambda-2 n+1)^{T}, & \text { else if } m>0 \text { and } \tilde{x}-\tilde{y}<0 \text { and } r_{x}-r_{y} \leq \tilde{x}-\tilde{y} ; \\ (2 n+1, \lambda-2 n-1)^{T}, & \text { else. }\end{cases}
$$

Here Quant[.] is the quantization function, i.e., if $x<0$, Quant $[x]=0$; if $x>\lambda$, Quant $[x]=\lambda$; if $0 \leq x \leq \lambda$, Quant $[x]=\lfloor x+0.5\rfloor$. Specifically for the identity channel matrix, the performance of ZF-MD detection is the exactly same as that of joint maximum likelihood detection.

The proof of Theorem 2 is postponed to the Appendix.

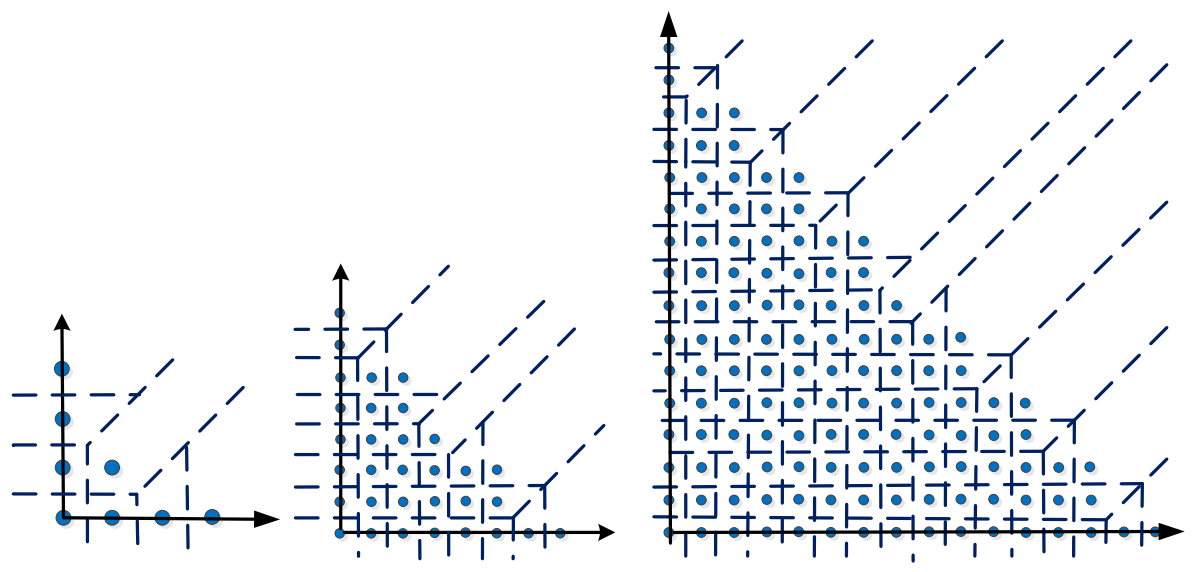

(a) $\mathrm{L}=8$

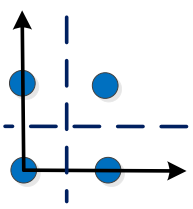

(a) $\mathrm{L}=4$

(b) $\mathrm{L}=32$

(a) $L=8,32,128$

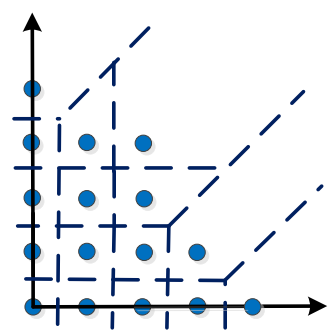

(b) $\mathrm{L}=16$

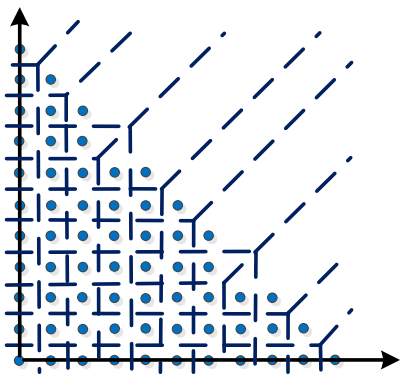

(c) $\mathrm{L}=64$

(b) $L=4,16,64$

Fig. 3. Decision regions of SCC.

From Theorem 2, we can draw the decision regions of SCC with two transmitters as shown in Fig. 3(a). In spite of the fact that we present a fast decoding algorithm for collaborative 
constellations only when $L=8,32,128, \ldots$ in Theorem 2 , the corresponding fast decoding algorithms can be developed in a straightforward manner for $L=4,16,64, \ldots$, respectively, with a minor difference just being some modifications of the points on the diagonal line. The decision regions of SCC when $L=4,16,64, \ldots$ are shown in Fig. 3(b) and we omit their fast algorithms here because of page space limitation.

\section{Complexity Analysis}

In the next, we compare the decoding complexity of traditional ML algorithm and the fast algorithm presented in this paper for SCC.

From (6), we can observe that the traditional ML decoding algorithm needs $2 L$ subtractions, $L$ multiplications to compute $\|\mathbf{r}-\mathbf{H s}\|^{2}, \mathbf{s} \in \Omega$, and needs $\log _{2} L$ subtractions to obtain the minimum value. Therefore, when the constellation size is $L$, the decoding complexity of traditional ML algorithm is $2 L+\log _{2} L$ subtractions and $L$ multiplications.

For the fast detection of SCC presented in this paper, it includes the following three steps:

1) Step 1 is the quantization to obtain $\tilde{x}=\operatorname{Quant}\left[r_{x}\right], \tilde{y}=\operatorname{Quant}\left[r_{y}\right]$. The two symbols are independent in $\Omega_{+}$, and the decoding complexity is determined by the square root of the cardinality of the constellation $\Omega_{+}$, so the decoding needs $2(\lambda+1) \approx 2 \sqrt{2 L}$ comparisons/subtractions.

2) Step 2 is to judge whether $(\tilde{x}, \tilde{y})^{T} \in \Omega$ by calculating $\tilde{x}+\tilde{y}$. If $\tilde{x}+\tilde{y}<\lambda$; if $\tilde{x}+$ $\tilde{y}=\lambda, \bmod (\lambda-|\tilde{x}-\tilde{y}|, 4)=0, \hat{\mathbf{s}}=(\tilde{x}, \tilde{y})^{T}$; otherwise, it will need Step 3. The decoding complexity of Step 2 is 3 addition/comparison operations, and it is robust to the constellation size. Furthermore, the possibility that the decoding needs Step 3 is about 0.5.

$3)$ Step 3 is to obtain $\hat{\mathbf{s}}$ based on the location of $(\tilde{x}, \tilde{y})$. Step 3 needs some operations, such as to compute $\frac{\lambda-|\tilde{x}-\tilde{y}|}{4}$, to judge $r_{x}-r_{y} \geq 0, r_{x}-r_{y} \leq \tilde{x}-\tilde{y}$, and to obtain $(\lambda-2 n-1,2 n+1)^{T}$. On the average, Step 3 needs 2 multiplications, and 9 addition/subtraction/comparison operations, which is also robust to the constellation size.

Algorithm complexity comparisons for traditional and fast decoding with different constellation sizes are listed in Table. V, where the number of additions/subtractions/comparisons is denoted as $N_{a}$ and the number of multiplications is denoted as $N_{m}$. From Table. V, we observe that the complexity of fast decoding algorithm for SCC is decreased significantly, and is almost linear with respect to the square root of the cardinality of the constellation $L$. 
TABLE V

ALGORITHM COMPLEXITY COMPARISON FOR TRADITIONAL ML AND FAST DECODING WITH DIFFERENT COLLABORATIVE CONSTELLATION SIZES

\begin{tabular}{|c||c|c|c|c|c|c|}
\hline \hline Constellation size $L$ & 8 & 16 & 32 & 64 & 128 & 256 \\
\hline \hline Decoding complexity & $N_{a}=19$ & $N_{a}=36$ & $N_{a}=69$ & $N_{a}=134$ & $N_{a}=263$ & $N_{a}=520$ \\
of traditional ML & $N_{m}=8$ & $N_{m}=16$ & $N_{m}=32$ & $N_{m}=64$ & $N_{m}=128$ & $N_{m}=256$ \\
\hline Decoding complexity & $N_{a}=16$ & $N_{a}=20$ & $N_{a}=24$ & $N_{a}=32$ & $N_{a}=40$ & $N_{a}=54$ \\
of fast decoding & $N_{m}=1$ & $N_{m}=1$ & $N_{m}=1$ & $N_{m}=1$ & $N_{m}=1$ & $N_{m}=1$ \\
\hline \hline
\end{tabular}

\section{Symbol Error Rate of SCC}

Based on the decision regions of SCC, we can analysis the symbol error rate of SCC in AWGN channel.

Theorem 3: When $L=8,32,128, \ldots$, the SER of $2 \times 2$ MIMO SCC is

$$
\begin{aligned}
P_{s} & =\frac{8 L-7 \sqrt{2 L}+2}{2 L} Q\left(\frac{d}{2 \sigma}\right)+\frac{\sqrt{2 L}-2}{L} Q\left(\frac{3 d}{2 \sigma}\right) \\
& -\frac{8 L-13 \sqrt{2 L}+10}{2 L} Q^{2}\left(\frac{d}{2 \sigma}\right)-\frac{3(\sqrt{2 L}-2)}{2 L} Q\left(\frac{d}{2 \sigma}\right) Q\left(\frac{3 d}{2 \sigma}\right),
\end{aligned}
$$

where $d=1 / P(\boldsymbol{\Omega})$.

The proof of Theorem 3 is postponed to the Appendix B.

From Fig. 3(b), it can be seen that, when $L=4,16,64,256$, the collaborative constellations and their decision regions have not a unified expression. Hence, following the same way, we can analysis the SER for $L=4,16,64,256$, respectively, and we omit them here because of page space limitation.

\section{BIT MAPPING OF SCC}

In general, Gray mapping is a preferred way to reduce the BER of communication systems. Therefore, we adopt Gray mapping for SCC constellations so as to make the different bits of adjacent points as small as possible.

When $L=4,16,64$, the bit mappings of SCC we proposed are shown in Fig. 4(a), where the bit mappings of SCC can be viewed as a transformation from those of square quadrature amplitude modulation (QAM) constellations, such as 4-QAM, 16-QAM, and 64-QAM. We will 
observe that most of adjacent points in SCC differ in one bit, but few of adjacent points differ in two bits.

When $L=8,32,128$, the bit mappings of SCC are shown in Fig. 4(b). We will observe that the bit mappings of SCC can be modified from those of rectangular QAM constellations, such as $2 \times 4$-QAM, $4 \times 8$-QAM, and $8 \times 16$-QAM.

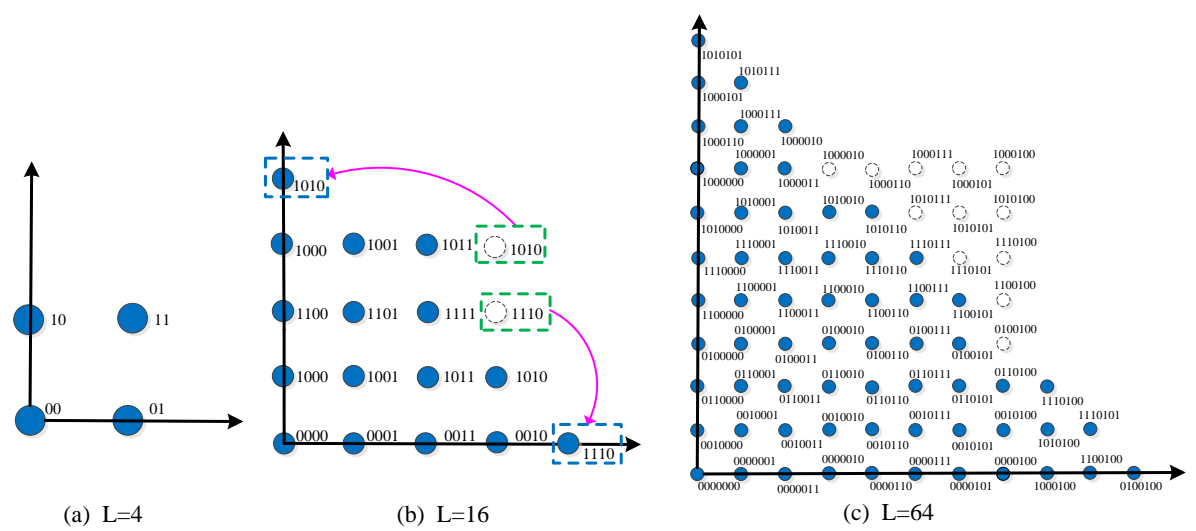

(a) $L=4,16,64$.

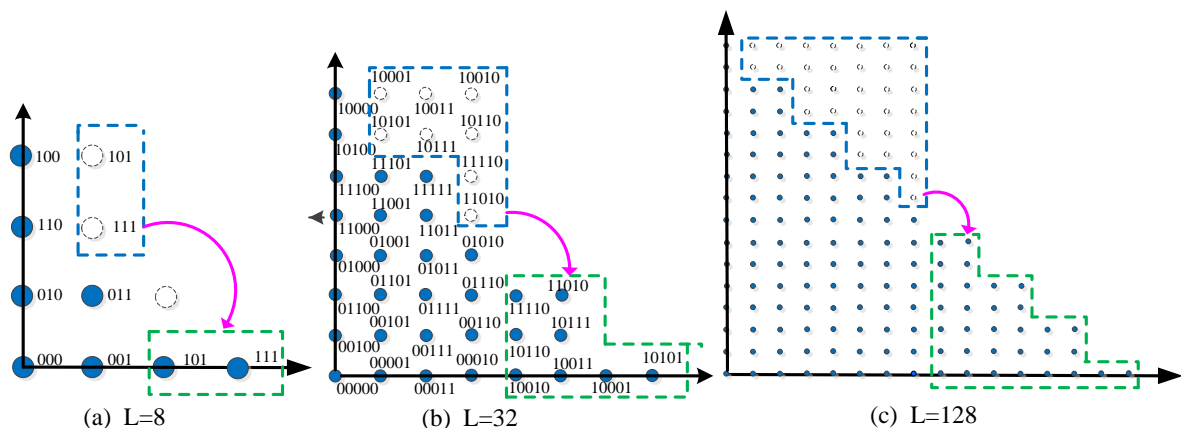

(b) $L=8,32,128$.

Fig. 4. Bit mapping of SCC.

\section{Simulation Results}

In the following, we consider a MIMO-VLC indoor scenario and carry out some computer simulations to verify our fast decoding algorithm and to compare the average BER of our proposed SCC scheme with those of other MIMO-VLC schemes such as RC, SM and SMP.

In the simulations, the optical power of the transmitted vector $\mathbf{S}$ is normalized as $\frac{P_{T}}{P(\Omega)} \mathbf{S}$. Therefore, the transmission optical power of the $M$ LEDs is $\frac{P_{T} \mathbb{E}\left[\|\mathbf{s}\|_{1}\right]}{P(\boldsymbol{\Omega})}=M P_{T}$ in total. Thus, the 
average signal-to-noise ratio ( $\mathrm{SNR}$ ) of each LED is defined as $\mathrm{SNR}=\frac{P_{T}}{\sigma^{2}}$. Here, we assume that $\sigma^{2}$ is fixed. When we vary the transmit optical power $P_{T}, \mathrm{SNR}$ is varied accordingly. Details of the MIMO-VLC system are given as follows. The LEDs are directed toward the floor and the PDs are directed toward the ceiling in a $4 m \times 4 m \times 3 m$ room. $A=1 \mathrm{~cm}^{2}, \Phi_{1 / 2}=\Psi_{1 / 2}=30^{\circ}$.

\section{A. Numerical Verify for Symbol Error Rate Analysis}

Theorem 3 presents a theoretical analysis formula of the symbol error rate of $2 \times 2 \mathrm{MIMO}$ SCC in AWGN channel. We can use numerical simulation to verify whether it is correct. Fig. 5 demonstrates the SER performance comparisons of numerical simulations and theoretical analysis formulas. This verifies that theoretical analysis formulas are correct.

\section{B. Error Performance Comparison for Different Decoding Algorithms}

Here, we compare the decoding performance of traditional ML algorithm and the fast decoding algorithm we proposed in this paper.

We consider a $2 \times 2$ MIMO system. The location of LEDs and PDs are fixed. The two PDs are located at $(x, y, z)=(0.1 \mathrm{~m}, 0,0.75 \mathrm{~m}),(0,0.1 \mathrm{~m}, 0.75 \mathrm{~m})$. The two LEDs are placed at $(d, 0,2.50 \mathrm{~m})$ and $(0, d, 2.5 \mathrm{~m})$. For $d=0.6 \mathrm{~m}$ and $0.4 \mathrm{~m}$, the corresponding channel matrixes are as followings:

$$
\begin{aligned}
& \mathbf{H}_{1}=2.1820 \times 10^{-4}\left(\begin{array}{cc}
0.2784 & 0 \\
0 & 0.2784
\end{array}\right), \\
& \mathbf{H}_{2}=2.1820 \times 10^{-4}\left(\begin{array}{ll}
0.4307 & 0.2416 \\
0.2416 & 0.4307
\end{array}\right) .
\end{aligned}
$$

We can observe that the channel correlations of $\mathbf{H}_{1}$ and $\mathbf{H}_{2}$ are different. $\mathbf{H}_{1}$ is an identity matrix, but the condition number of matrix $\mathbf{H}_{2}$ is 3.56.

When the channel matrix is $\mathbf{H}_{1}$, Fig. 6(a) demonstrates the performance of the two algorithms for $L=8,32,128,512$. It can be seen the performance of fast decoding algorithm is exactly same as that of traditional ML algorithm, while the complexity of fast decoding is almost linear. This verifies that the performance of the fast decoding algorithm is equal to that of traditional ML algorithm when the channel matrix is an identity matrix. 


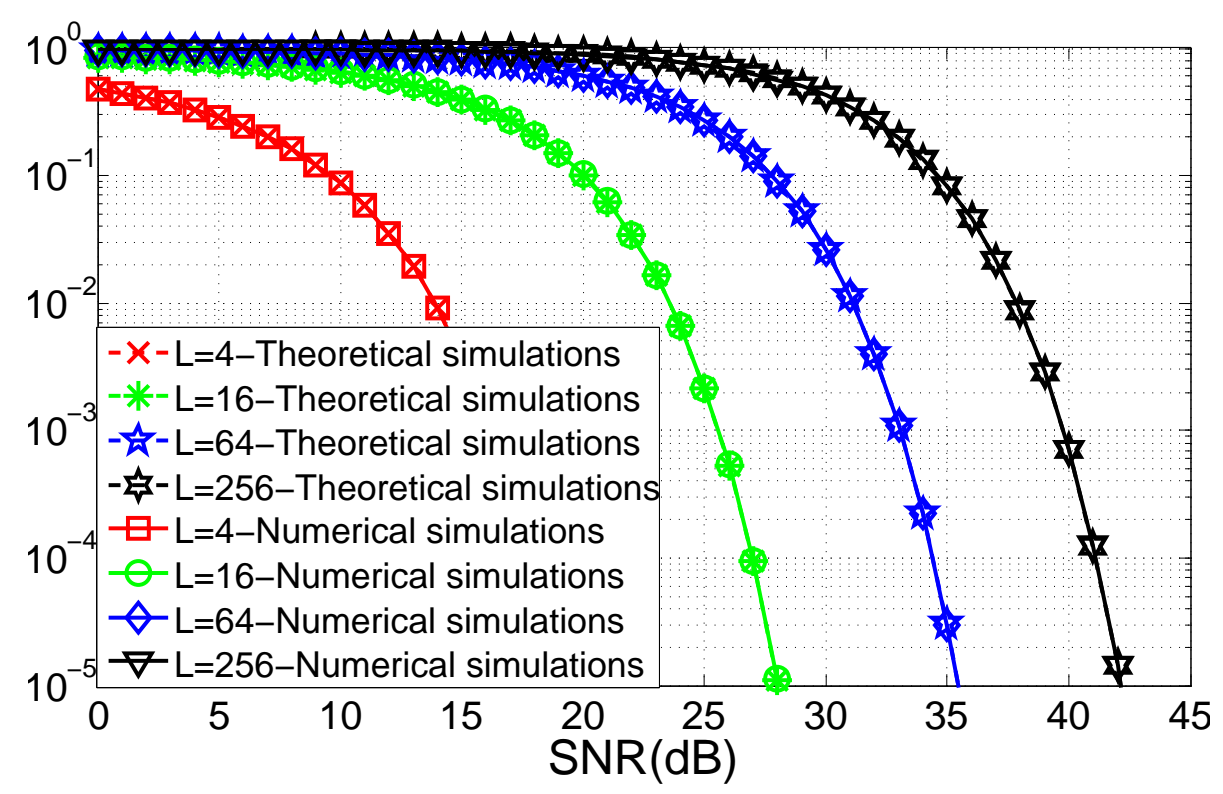

(a) $L=4,16,64,256$.

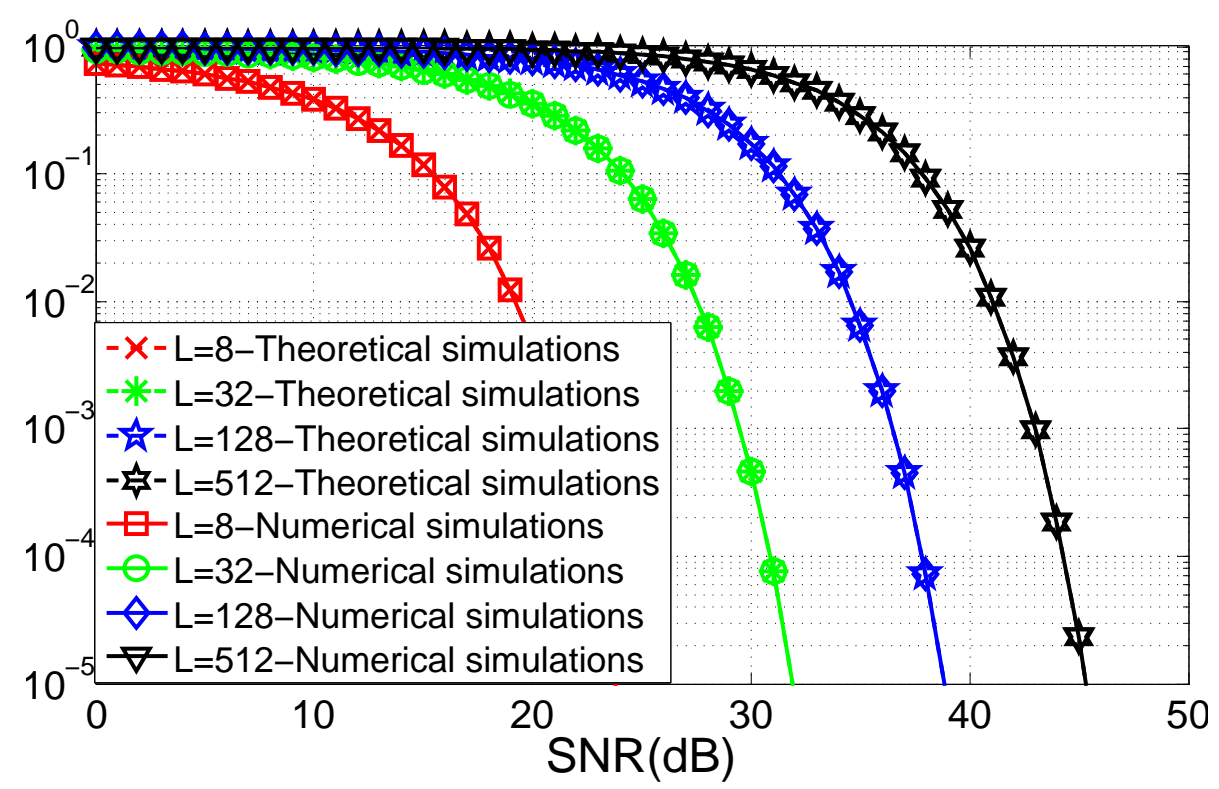

(b) $L=8,32,128,512$.

Fig. 5. SER performance comparisons of numerical simulations and theoretical analysis formulas. 


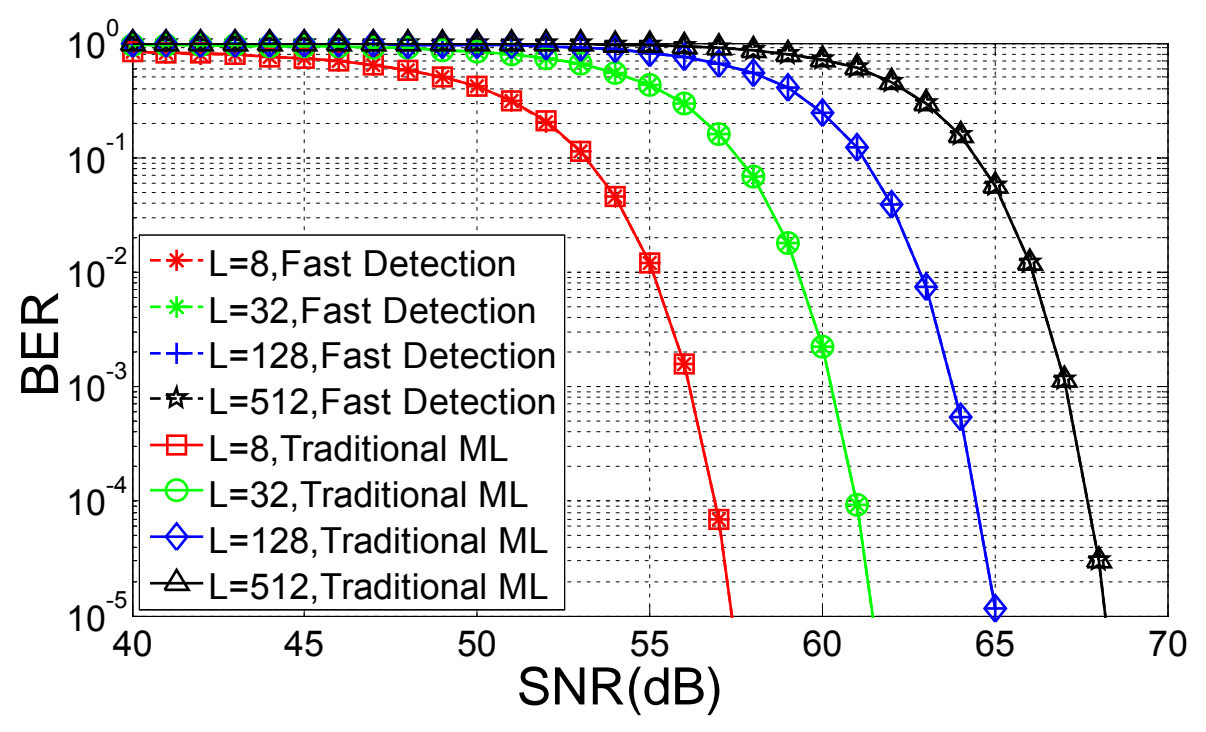

(a) $L=8,32,128,512$.

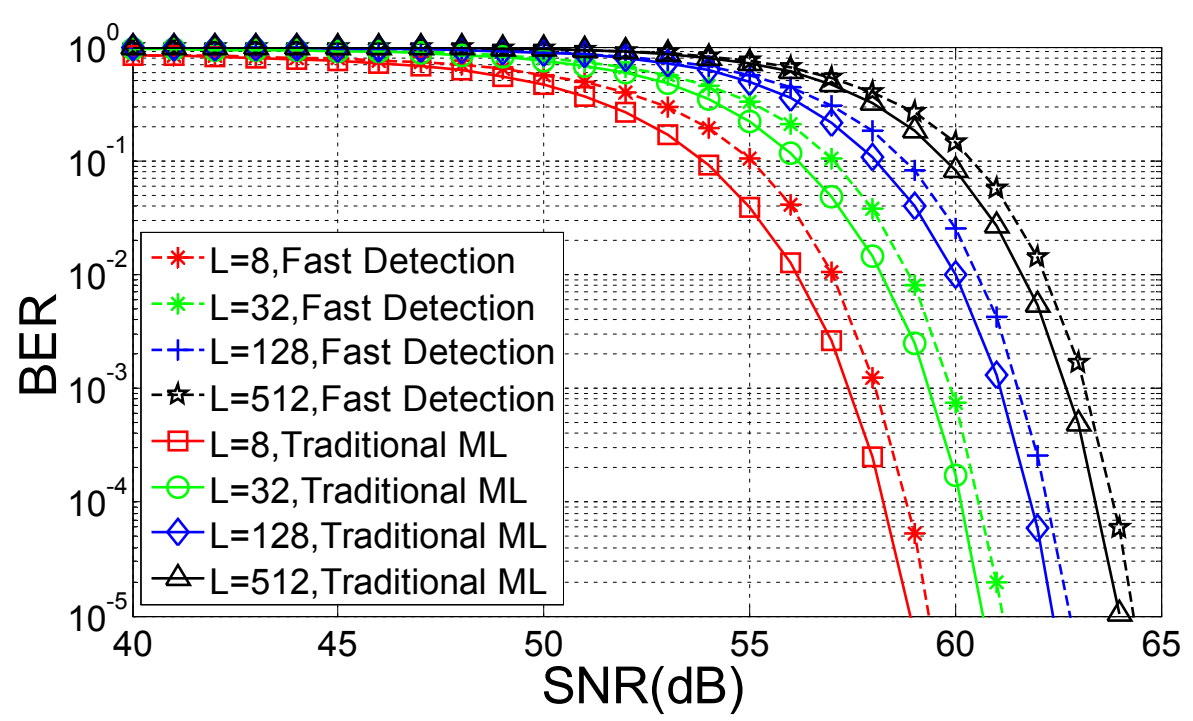

(b) $L=8,32,128,512$.

Fig. 6. Decoding performance comparisons of traditional ML algorithm and the fast decoding algorithm.

When the channel matrix is $\mathbf{H}_{2}$, Fig. 6(b) demonstrates the performance of the two algorithms for $L=8,32,128,512$. It can be seen that the performance of fast decoding algorithm is worse than that of traditional ML Algorithm about $0.5 \sim 0.8 \mathrm{~dB}$. Similar to the RF communications, the performance loss is caused by the $\mathrm{ZF}$ detector used in (7). 


\section{Error Performance Comparison for Different MIMO Schemes}

Here, we compare the BER of SCC scheme with those of RC, SM and SMP. To compare their performance under different channel correlations, we assume that the location of LEDs are fixed while the receiver with multiple PDs randomly moves around the room.

First, we consider a $2 \times 2$ MIMO system. We assume that the two LEDs are located at $(2 m, 1.8 m, 2.5 m)$ and $(2 m, 2.2 m, 2.5 m)$. The two PDs are placed at $(X m,(Y-0.4) m, 0.75 m)$ and $(X m,(Y+0.4) m, 0.75 m)$, and the values of $X$ and $Y$ are independently and equally-likely changed between 0 and 4 except for the case that the channel matrix is zero, so the channel states are almost ergodic during the communication. We compare the BER performance of RC, SM, SMP and SCC when $L=4,8,16,32$ and $L=64,128,256,512$, as shown in Fig. 7 (a) and Fig. 7 (b), respectively. It can be seen that the performance of SCC is the best when $L$ is larger than 64 in the high SNR region, however, $\mathrm{RC}$ is the best when $L$ is small. In fact, the Euclidean distance of a constellation and the channel correlation affect the performance of MIMO VLC simultaneously. When $L$ is small, the Euclidean distance gap between RC and SCC is small. Therefore, we can conclude that channel correlation plays a major role when $L$ is small, but the Euclidean distance plays a major role when $L$ is large. Furthermore, SM is the best in the low SNR region where BER is above $10^{-3}$. The performance gap between SCC and SMP is about $0.5 \sim 0.8 \mathrm{~dB}$.

Next, we consider a $3 \times 3$ MIMO system. The three LEDs are located at $(2 \mathrm{~m}, 1.6 \mathrm{~m}, 2.5 \mathrm{~m})$, $(2 m, 2 m, 2.5 m)$ and $(2 m, 2.4 m, 2.5 m)$. The three PDs are placed at $(X m,(Y-0.4) m, 0.75 m)$, $(X m, Y m, 0.75 m)$ and $(X m,(Y+0.4) m, 0.75 m)$, and the values of $X$ and $Y$ are independently and equally-likely changed between 0 and 4 except for the case that the channel matrix is zero. The BER comparisons of RC, SM, SMP and SCC are shown in Fig. 8. It can be seen that the performance of SCC is also the best. SCC achieves $3 \sim 5 \mathrm{~dB}$ gain than SM when the error rate is $10^{-5}$.

Finally, we consider a $4 \times 4$ MIMO system. The four LEDs are located at $(1 m, 1 m, 2.5 m),(3 m, 1 m, 2.5 m),(1 m, 3 m, 2.5 m)$ and $(3 m, 3 m, 2.5 m)$. The four PDs are placed at $(X m, X m, 0.75 m),(X m, Y m, 0.75 m),(Y m, X m, 0.75 m)$ and $(Y m, Y m, 0.75 m)$, where the values of $X$ is independently and equally-likely changed between 0 and 2, and $Y$ is independently and equally-likely changed between 2 and 4 except for the case that the channel matrix is zero. 

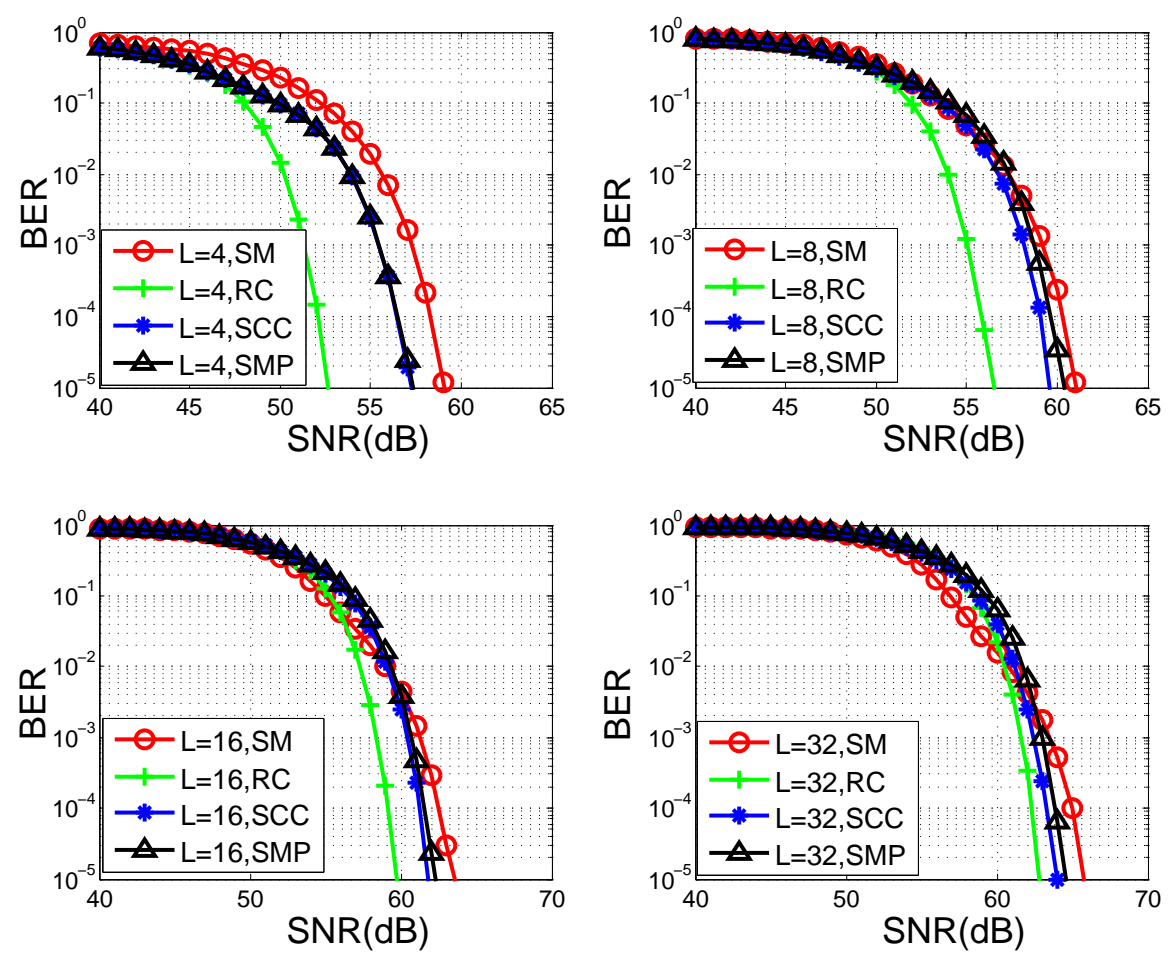

(a) $L=4,8,16,32$.
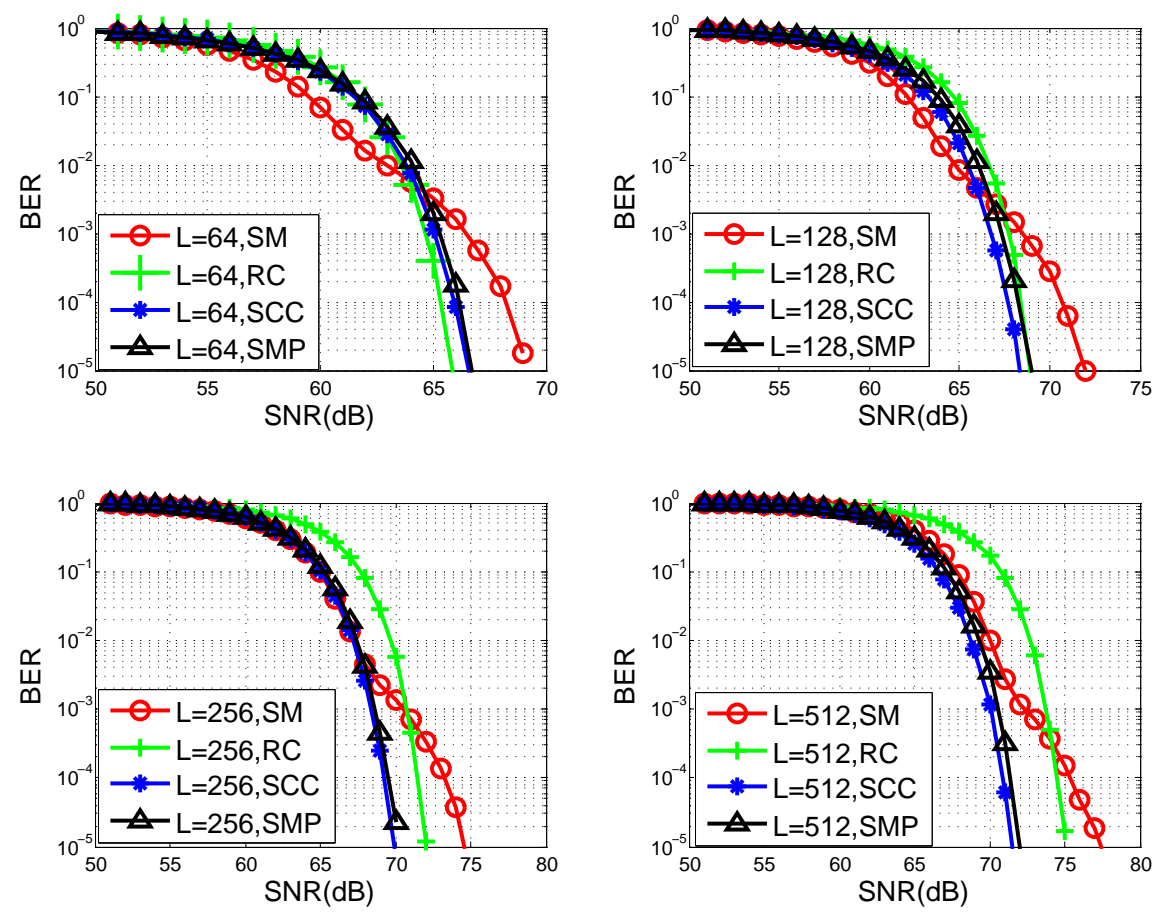

(b) $L=64,128,256,512$.

Fig. 7. BER comparisons of SM, RC, SMP and SCC in a $2 \times 2$ MIMO-VLC system with the receiver location varying within the room. 

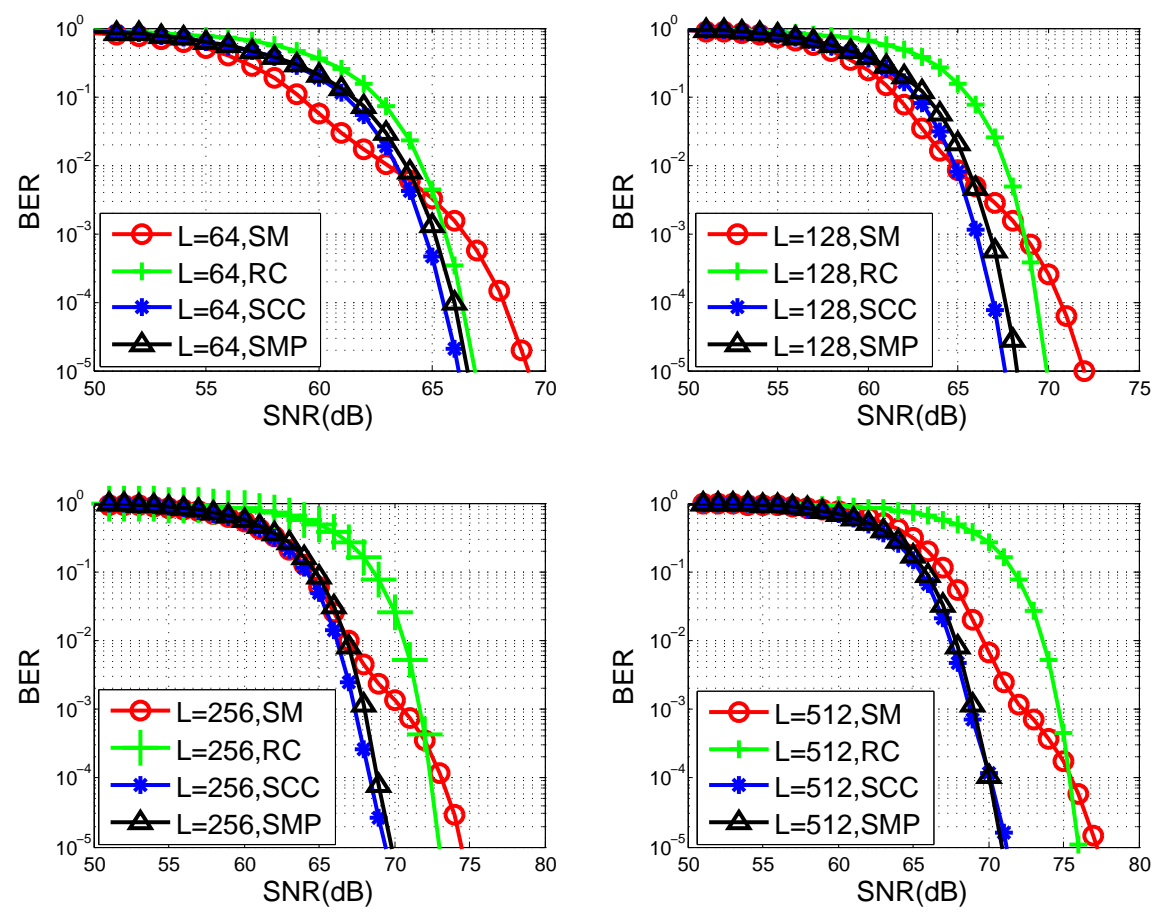

Fig. 8. BER comparisons of SM, RC, SMP and SCC in a $3 \times 3$ MIMO-VLC system with the receiver location varying within the room.

The BER comparisons of RC, SM, SMP and SCC are shown in Fig. 9. It can be seen that the performance of SCC is still the best. Even when the constellation size is very large, SCC is better than SMP, which is the best MIMO technique for low correlation channel in literature [10].

Above all, from Fig. 7, Fig. 8, and Fig. 9, we can observe that SCC is the best and performs the same in all cases. RC performs well when the constellation size is relatively small in $2 \times 2$ MIMO case, while the gap between RC and SCC is about $3-8 \mathrm{~dB}$ when the constellation size is large. SMP is the second best one, and the performance gap between SMP and SCC is about $0.5-1.5 \mathrm{~dB}$. Furthermore, we will notice that both the Euclidean distance of a constellation and channel correlation affect the performance of MIMO VLC, but SCC is the best when $L$ is large.

\section{CONCLUSION}

In this paper, we considered SCC design for indoor MIMO VLC systems. We presented a unified SCC structure for $2 \times 2$ MIMO VLC systems, and extended it to larger MIMO VLC 

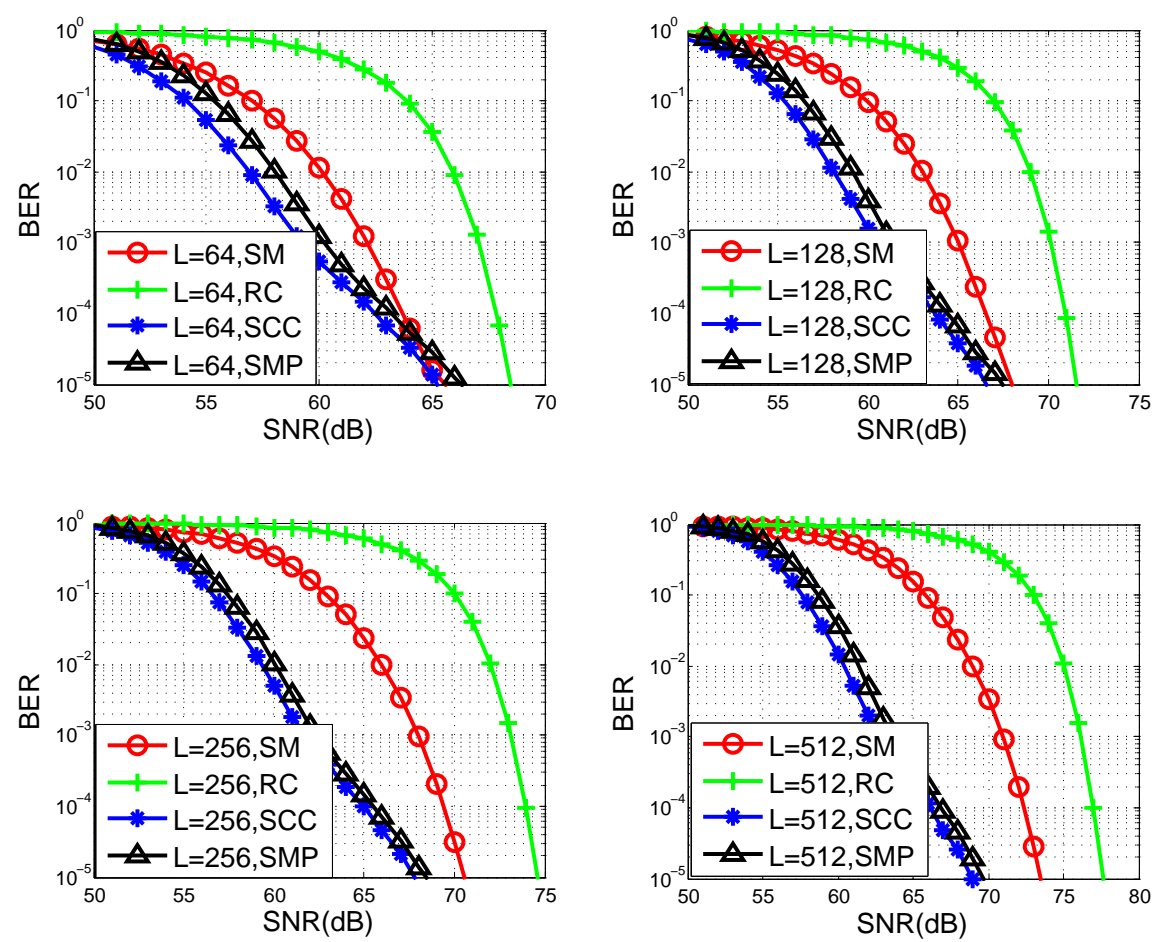

Fig. 9. BER comparisons of SM, RC, SMP and SCC in a $4 \times 4$ MIMO-VLC system with the receiver location varying within the room.

systems with more transceivers. We have developed a fast decoding algorithm for SCC whose complexity is almost linear in terms of the square root of the cardinality of the constellation. In addition, bit mappings for SCC are proposed. Computer simulation results demonstrated that the fast detection algorithm is effective, and the performance of SCC is better than RC, SM and SMP for indoor mobile channels in general.

\section{ACKNOWLEDGEMENTS}

The authors wish to thank the anonymous reviewers for their valuable suggestions.

\section{APPENDIX}

\section{A. Proof of Theorem 2}

When $L=8,32,128, \ldots$, the optimal collaborative constellation $\Omega$ is described in Lemma 2 . Let $\Omega_{+}=\left\{\mathbf{s}=(x, y)^{T}: 0 \leq x \leq \lambda, 0 \leq y \leq \lambda\right\}$, we can observe that $\boldsymbol{\Omega}_{+}$is a square constellation with the size of $(\lambda+1)^{2}$. Let $\boldsymbol{\Omega}_{-}=\boldsymbol{\Omega}_{+}-\boldsymbol{\Omega}=\left\{\mathbf{s}=(x, y)^{T}: \mathbf{s} \in \boldsymbol{\Omega}_{+}, \mathbf{s} \notin \boldsymbol{\Omega}\right\}$, then the 
points in constellation $\Omega_{-}$are the dotted circles and the points in constellation $\Omega$ are the solid line circles, as shown in Fig. 10.

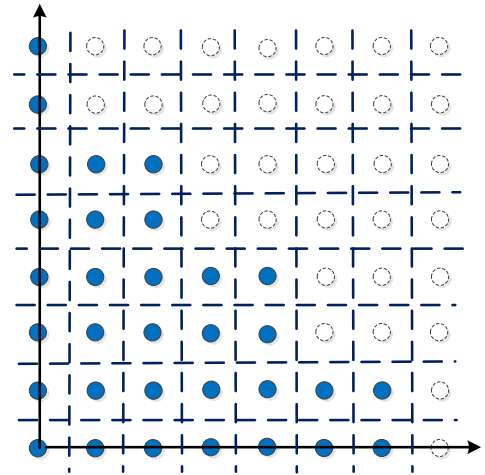

(a)

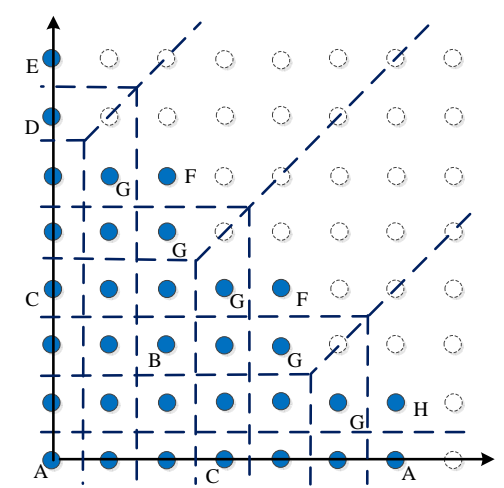

(b)

Fig. 10. The diagrammatic sketch of decision regions of SCC when $L=2^{2 q+1}, q=1,2, \ldots$. As an example, $q=2$ here.

Since $\Omega_{+}$is a square constellation, the decision regions of $\Omega_{+}$are shown in Fig. 10(a), and we can obtain that

$$
\begin{aligned}
\tilde{x} & =\operatorname{Quant}\left[r_{x}\right]=\arg \min _{0 \leq x \leq \lambda}\left|r_{x}-x\right|^{2}, \\
\tilde{y} & =\operatorname{Quant}\left[r_{y}\right]=\arg \min _{0 \leq y \leq \lambda}\left|r_{y}-y\right|^{2}, \\
\tilde{\mathbf{s}}=(\tilde{x}, \tilde{y})^{T} & =\arg \min _{\mathbf{s} \in \boldsymbol{\Omega}_{+}}\left(\left|r_{x}-x\right|^{2}+\left|r_{y}-y\right|^{2}\right) .
\end{aligned}
$$

Since $\Omega_{+}=\Omega+\Omega_{-}$, $\tilde{\mathrm{s}}$ can be rewritten as

$$
\begin{array}{r}
\tilde{\mathbf{s}}=\arg \min \left(\min _{\mathbf{s} \in \boldsymbol{\Omega}}\left(\left|r_{x}-x\right|^{2}+\left|r_{y}-y\right|^{2}\right),\right. \\
\left.\min _{\mathbf{s} \in \boldsymbol{\Omega}-}\left(\left|r_{x}-x\right|^{2}+\left|r_{y}-y\right|^{2}\right)\right) .
\end{array}
$$

However, the estimation vector $\hat{\mathbf{s}}=(\hat{x}, \hat{y})^{T}$ of $\mathbf{s}$ is

$$
\hat{\mathbf{s}}=\arg \min _{\mathbf{s} \in \mathbf{\Omega}}\left(\left|r_{x}-x\right|^{2}+\left|r_{y}-y\right|^{2}\right) .
$$

Therefore, we need to establish a relation between $\tilde{\mathbf{s}}$ and $\hat{\mathbf{s}}$ in the following.

Case 1: $\tilde{x}+\tilde{y}<\lambda$, then $\tilde{\mathbf{s}} \in \Omega$. From (13) and (14), we can obtain that $\hat{\mathbf{s}}=\tilde{\mathbf{s}}$.

Case 2: $\tilde{x}+\tilde{y}=\lambda$,

1) If $\bmod (\lambda-|\tilde{x}-\tilde{y}|, 4)=0$, then $\tilde{x}+\tilde{y}=\lambda, \lambda-|\tilde{x}-\tilde{y}|=4 k, k$ is an nonnegative integer. When $\tilde{x} \geq \tilde{y}, y=2 k, \tilde{x}=\lambda-2 k, k<\lambda / 2$; when $\tilde{x}<\tilde{y}, \tilde{x}=2 k, \tilde{y}=\lambda-2 k, k<\lambda / 2$. Therefore, $\tilde{\mathbf{s}} \in \Omega, \hat{\mathbf{s}}=\tilde{\mathbf{s}}$;

2) If $\bmod (\lambda-|\tilde{x}-\tilde{y}|, 4)>0$, then $\tilde{\mathbf{s}} \notin \Omega$. 
Let $r_{x}=\tilde{x}+\delta_{x}, r_{y}=\tilde{y}+\delta_{y},\left|\delta_{x}\right| \leq 0.5,\left|\delta_{y}\right| \leq 0.5$, then $\hat{\mathbf{s}}=\arg \min _{\mathbf{s} \in \boldsymbol{\Omega}}\left(\left|\tilde{x}+\delta_{x}-x\right|^{2}+\right.$ $\left.\left|\tilde{y}+\delta_{y}-y\right|^{2}\right)=\left[(\tilde{x}-x)^{2}+(\tilde{y}-y)^{2}\right]+2\left[\delta_{x}(\tilde{x}-x)+\delta_{y}(\tilde{y}-y)\right]+\delta_{x}^{2}+\delta_{y}^{2}$. Since $\tilde{x} \geq x$, $\tilde{y} \geq y$, we can obtain that $\min \left[(\tilde{x}-x)^{2}+(\tilde{y}-y)\right]^{2}=1$, where $\tilde{x}-x=1, \tilde{y}-y=0$, or $\tilde{x}-x=0, \tilde{y}-y=1$. Furthermore, $\min 2\left[\delta_{x}(\tilde{x}-x)+\delta_{y}(\tilde{y}-y)\right]$ is equal to $2 \delta_{x}$ if $\delta_{x} \leq \delta_{y}$, or $2 \delta_{y}$ if $\delta_{x}>\delta_{y}$. Therefore, we can obtain that if $r_{x}-r_{y} \leq \tilde{x}-\tilde{y}, \hat{\mathbf{s}}=(\tilde{x}-1, \tilde{y})^{T}$; if $r_{x}-r_{y}>\tilde{x}-\tilde{y}, \hat{\mathbf{s}}=(\tilde{x}, \tilde{y}-1)$.

Case 3: $\tilde{x}+\tilde{y}>\lambda$.

Since $\tilde{x} \geq x, \tilde{y} \geq y,\left[(\tilde{x}-x)^{2}+(\tilde{y}-y)\right]^{2} \geq 2(\tilde{x}-x)(\tilde{y}-y)$, the condition which makes $(\tilde{x}-x)^{2}+(\tilde{y}-y)^{2}$ achieve the minimum value is $\tilde{x}-x=\tilde{y}-y$, i.e., $x-y=\tilde{x}-\tilde{y}, x+y=\lambda$. Let $\delta_{0}=\min \left(\delta_{x}, \delta_{y}\right)$, then $2\left[\delta_{x}(\tilde{x}-x)+\delta_{y}(\tilde{y}-y)\right] \geq 2 \delta_{0}(\tilde{x}+\tilde{y}-x-y)$, and the condition which makes $2\left[\delta_{x}(\tilde{x}-x)+\delta_{y}(\tilde{y}-y)\right]$ achieve the minimum value is $x+y=\lambda$. Therefore, we can obtain that $x-y=\tilde{x}-\tilde{y}, x+y=\lambda, x=(\lambda+\tilde{x}-\tilde{y}) / 2, y=(\lambda-\tilde{x}+\tilde{y}) / 2$. Let $m=\bmod (\lambda-|\tilde{x}-\tilde{y}|, 4), n=\left\lfloor\frac{\lambda-|\tilde{x}-\tilde{y}|}{4}\right\rfloor$, then we discuss as follows:

1) If $m=0, \tilde{x}<\tilde{y}$, we can observe that $(\lambda-2 n, 2 n) \in \Omega$, then $\hat{\mathbf{s}}=(\lambda-2 n, 2 n)^{T}$.

2) If $m>0, \tilde{x}<\tilde{y}$, we can observe that $(\lambda-2 n, 2 n) \notin \Omega$, thus $\hat{\mathbf{s}}=(\lambda-2 n-1,2 n)^{T}$, or $\hat{\mathbf{s}}=(\lambda-2 n, 2 n-1)^{T}$. We can obtain that if $\delta_{x} \leq \delta_{y}$, i.e., $r_{x}-r_{y} \leq \tilde{x}-\tilde{y}, \hat{\mathbf{s}}=$ $(\lambda-2 n-1,2 n+1)^{T}$; if $r_{x}-r_{y}>\tilde{x}-\tilde{y}, \hat{\mathbf{s}}=(\lambda-2 n+1,2 n-1)^{T}$.

3) If $m=0, \tilde{x} \geq \tilde{y}$, we can observe that $(2 n, \lambda-2 n) \in \Omega$, then $\hat{\mathbf{s}}=(2 n, \lambda-2 n)^{T}$.

4) If $m>0, \tilde{x} \geq \tilde{y}$, we can observe that $(2 n, \lambda-2 n) \notin \Omega$, thus $\hat{\mathbf{s}}=(2 n+1, \lambda-2 n-1)^{T}$, or $\hat{\mathbf{s}}=(2 n-1, \lambda-2 n+1)^{T}$. We can obtain that if $\delta_{x} \leq \delta_{y}$, i.e., $r_{x}-r_{y} \leq \tilde{x}-\tilde{y}$, $\hat{\mathbf{s}}=(2 n+1, \lambda-2 n-1)^{T}$; if $r_{x}-r_{y}>\tilde{x}-\tilde{y}, \hat{\mathbf{s}}=(2 n-1, \lambda-2 n+1)^{T}$.

When $\mathbf{H}=\mathbf{I}, \tilde{\mathbf{r}}=\mathbf{s}+\mathbf{n}$, so the noise is Gaussian noise after ZF detection. In the AWGN channel, minimum distance based receiver is equal to JML receiver. Therefore, when the channel matrix is an identity matrix, the performance of ZF-MD detection is the same as that of JML detection. This completes the proof of Theorem 2.

\section{B. Proof of Theorem 3}

PROOF: When $L=2^{2 q+1}, q=1,2, \ldots$, the diagrammatic sketch of decision regions of SCC is shown in Fig. 10(b). We can observe that the decision regions can be classified into eight kinds, denoted as A,B,C,D,E,F,G,H, respectively. 
Case A: There are two symbols whose decision region is region A. The correct probability of each symbol is as follows:

$$
\begin{aligned}
P_{C A} & =\int_{-\infty}^{\frac{d}{2}} \frac{1}{\sqrt{2 \pi \sigma^{2}}} e^{-\frac{x^{2}}{2 \sigma^{2}}} d x \int_{-\infty}^{\frac{d}{2}} \frac{1}{\sqrt{2 \pi \sigma^{2}}} e^{-\frac{y^{2}}{2 \sigma^{2}}} d y \\
& =\left\{\int_{-\infty}^{\frac{d}{2 \sigma}} \frac{1}{\sqrt{2 \pi}} e^{-\frac{t^{2}}{2}} d t\right\}^{2}=\left\{1-Q\left(\frac{d}{2 \sigma}\right)\right\}^{2} .
\end{aligned}
$$

Case B: There are $(\lambda-2)(\lambda-3) / 2$ symbols whose decision region is region $\mathrm{B}$. The correct probability of each symbol is as follows:

$$
\begin{aligned}
P_{C B} & =\int_{a-d / 2}^{a+d / 2} \frac{1}{\sqrt{2 \pi \sigma^{2}}} e^{-\frac{(x-a)^{2}}{2 \sigma^{2}}} d x \int_{b-d / 2}^{b+d / 2} \frac{1}{\sqrt{2 \pi \sigma^{2}}} e^{-\frac{(y-b)^{2}}{2 \sigma^{2}}} d y \\
& =\int_{-d / 2}^{d / 2} \frac{1}{\sqrt{2 \pi \sigma^{2}}} e^{-\frac{x^{2}}{2 \sigma^{2}}} d x \int_{-d / 2}^{d / 2} \frac{1}{\sqrt{2 \pi \sigma^{2}}} e^{-\frac{y^{2}}{2 \sigma^{2}}} d y \\
& =\left\{1-2 Q\left(\frac{d}{2 \sigma}\right)\right\}^{2} .
\end{aligned}
$$

Case C: There are $2(\lambda-2)$ symbols whose decision region is region $\mathbf{C}$. The correct probability of each symbol is as follows:

$$
\begin{aligned}
P_{C C} & =\int_{m d-d / 2}^{m d+d / 2} \frac{1}{\sqrt{2 \pi \sigma^{2}}} e^{-\frac{(x-m d)^{2}}{2 \sigma^{2}}} d x \int_{-\infty}^{d / 2} \frac{1}{\sqrt{2 \pi \sigma^{2}}} e^{-\frac{y^{2}}{2 \sigma^{2}}} d y \\
& =\int_{-d / 2}^{d / 2} \frac{1}{\sqrt{2 \pi \sigma^{2}}} e^{-\frac{x^{2}}{2 \sigma^{2}}} d x \int_{-\infty}^{d / 2} \frac{1}{\sqrt{2 \pi \sigma^{2}}} e^{-\frac{y^{2}}{2 \sigma^{2}}} d y \\
& =\left\{1-Q\left(\frac{d}{2 \sigma}\right)\right\}\left\{1-2 Q\left(\frac{d}{2 \sigma}\right)\right\} .
\end{aligned}
$$

Case D: There is one symbol whose decision region is region D. The correct probability of the symbol is as follows:

$$
\begin{aligned}
P_{C D}= & \int_{-d / 2}^{d / 2} \frac{1}{\sqrt{2 \pi \sigma^{2}}} e^{-\frac{y^{2}}{2 \sigma^{2}}} d y \int_{-\infty}^{d / 2} \frac{1}{\sqrt{2 \pi \sigma^{2}}} e^{-\frac{x^{2}}{2 \sigma^{2}}} d x+ \\
& \int_{-d / 2}^{d / 2} \frac{1}{\sqrt{2 \pi \sigma^{2}}} e^{-\frac{y^{2}}{2 \sigma^{2}}} d y \int_{d / 2}^{y+d} \frac{1}{\sqrt{2 \pi \sigma^{2}}} e^{-\frac{x^{2}}{2 \sigma^{2}}} d x
\end{aligned}
$$

The corresponding decision region of the second item in (15) is not a regular domain of integration, as shown in Fig. 11(a), and we use a half of a square domain to approximate it. Thus, we can obtain

$$
\begin{aligned}
P_{C D} & \approx \int_{-d / 2}^{d / 2} \frac{1}{\sqrt{2 \pi \sigma^{2}}} e^{-\frac{y^{2}}{2 \sigma^{2}}} d y \int_{-\infty}^{d / 2} \frac{1}{\sqrt{2 \pi \sigma^{2}}} e^{-\frac{x^{2}}{2 \sigma^{2}}} d x \\
& +\frac{1}{2} \int_{-d / 2}^{d / 2} \frac{1}{\sqrt{2 \pi \sigma^{2}}} e^{-\frac{y^{2}}{2 \sigma^{2}}} d y \int_{d / 2}^{3 d / 2} \frac{1}{\sqrt{2 \pi \sigma^{2}}} e^{-\frac{x^{2}}{2 \sigma^{2}}} d x \\
& =1-\frac{5}{2} Q\left(\frac{d}{2 \sigma}\right)-\frac{1}{2} Q\left(\frac{3 d}{2 \sigma}\right)+Q^{2}\left(\frac{d}{2 \sigma}\right)+Q\left(\frac{d}{2 \sigma}\right) Q\left(\frac{3 d}{2 \sigma}\right)
\end{aligned}
$$



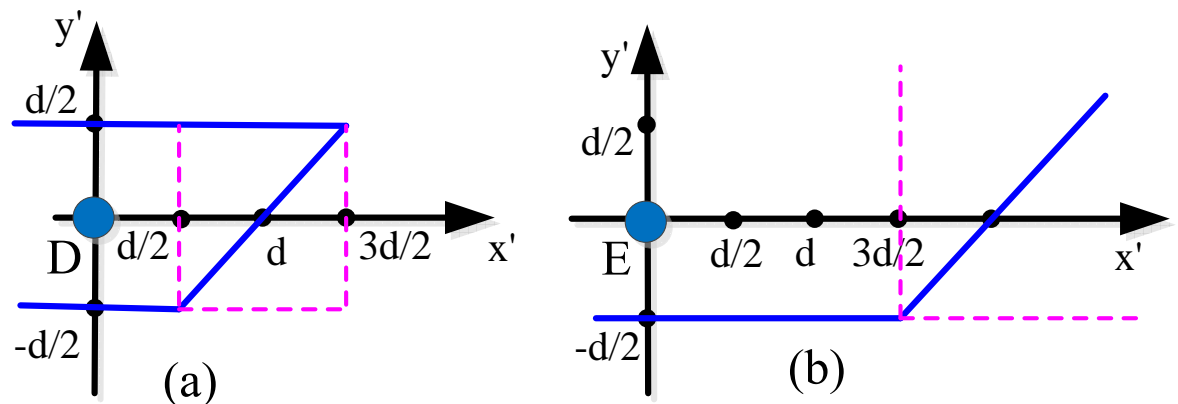

Fig. 11. The approximation of decision region.

Case E: There is one symbol whose decision region is region E. The correct probability of the symbol is as follows:

$$
\begin{aligned}
P_{C E} & \approx \int_{-d / 2}^{\infty} \frac{1}{\sqrt{2 \pi \sigma^{2}}} e^{-\frac{y^{2}}{2 \sigma^{2}}} d y \int_{-\infty}^{3 d / 2} \frac{1}{\sqrt{2 \pi \sigma^{2}}} e^{-\frac{x^{2}}{2 \sigma^{2}}} d x \\
& +\int_{-d / 2}^{\infty} \frac{1}{\sqrt{2 \pi \sigma^{2}}} e^{-\frac{y^{2}}{2 \sigma^{2}}} d y \int_{3 d / 2}^{y+2 d} \frac{1}{\sqrt{2 \pi \sigma^{2}}} e^{-\frac{x^{2}}{2 \sigma^{2}}} d x
\end{aligned}
$$

The corresponding decision region in (16) is not a regular domain of integration, as shown in Fig. 11(b), and we approximate it as follows

$$
\begin{aligned}
P_{C E} & \approx \int_{-d / 2}^{\infty} \frac{1}{\sqrt{2 \pi \sigma^{2}}} e^{-\frac{y^{2}}{2 \sigma^{2}}} d y \int_{-\infty}^{3 d / 2} \frac{1}{\sqrt{2 \pi \sigma^{2}}} e^{-\frac{x^{2}}{2 \sigma^{2}}} d x \\
& +\frac{1}{2} \int_{-d / 2}^{\infty} \frac{1}{\sqrt{2 \pi \sigma^{2}}} e^{-\frac{y^{2}}{2 \sigma^{2}}} d y \int_{3 d / 2}^{\infty} \frac{1}{\sqrt{2 \pi \sigma^{2}}} e^{-\frac{x^{2}}{2 \sigma^{2}}} d x \\
& =1-Q\left(\frac{d}{2 \sigma}\right)-\frac{1}{2} Q\left(\frac{3 d}{2 \sigma}\right)+\frac{1}{2} Q\left(\frac{d}{2 \sigma}\right) Q\left(\frac{3 d}{2 \sigma}\right) .
\end{aligned}
$$

Case F: There are $(\lambda-3) / 2$ symbols whose decision region is region $\mathrm{F}$, and the correct probability of each symbol is as follows:

$$
\begin{aligned}
P_{C F} & =\int_{-d / 2}^{3 d / 2} \frac{1}{\sqrt{2 \pi \sigma^{2}}} e^{-\frac{x^{2}}{2 \sigma^{2}}} d x \int_{-d / 2}^{x+2 d} \frac{1}{\sqrt{2 \pi \sigma^{2}}} e^{-\frac{y^{2}}{2 \sigma^{2}}} d y \\
& +\int_{3 d / 2}^{\infty} \frac{1}{\sqrt{2 \pi \sigma^{2}}} e^{-\frac{x^{2}}{2 \sigma^{2}}} d x \int_{x-2 d}^{x+2 d} \frac{1}{\sqrt{2 \pi \sigma^{2}}} e^{-\frac{y^{2}}{2 \sigma^{2}}} d y \\
& \approx \int_{-d / 2}^{\infty} \frac{1}{\sqrt{2 \pi \sigma^{2}}} e^{-\frac{x^{2}}{2 \sigma^{2}}} d x \int_{-d / 2}^{\infty} \frac{1}{\sqrt{2 \pi \sigma^{2}}} e^{-\frac{y^{2}}{2 \sigma^{2}}} d y \\
& -\frac{1}{2} \int_{-d / 2}^{\infty} \frac{1}{\sqrt{2 \pi \sigma^{2}}} e^{-\frac{x^{2}}{2 \sigma^{2}}} d x \int_{3 d / 2}^{\infty} \frac{1}{\sqrt{2 \pi \sigma^{2}}} e^{-\frac{y^{2}}{2 \sigma^{2}}} d y \\
& -\frac{1}{2} \int_{3 d / 2}^{\infty} \frac{1}{\sqrt{2 \pi \sigma^{2}}} e^{-\frac{x^{2}}{2 \sigma^{2}}} d x \int_{-d / 2}^{\infty} \frac{1}{\sqrt{2 \pi \sigma^{2}}} e^{-\frac{y^{2}}{2 \sigma^{2}}} d y \\
& =1-2 Q\left(\frac{d}{2 \sigma}\right)-Q\left(\frac{3 d}{2 \sigma}\right)+Q^{2}\left(\frac{d}{2 \sigma}\right)+Q\left(\frac{d}{2 \sigma}\right) Q\left(\frac{3 d}{2 \sigma}\right) .
\end{aligned}
$$


Case G: There are $\lambda-2$ symbols whose decision region is region $\mathrm{G}$, and the correct probability of each symbol is as follows:

$$
\begin{aligned}
P_{C G} & =\int_{-d / 2}^{d / 2} \frac{1}{\sqrt{2 \pi \sigma^{2}}} e^{-\frac{x^{2}}{2 \sigma^{2}}} d x \int_{-d / 2}^{x+d} \frac{1}{\sqrt{2 \pi \sigma^{2}}} e^{-\frac{y^{2}}{2 \sigma^{2}}} d y \\
& \approx \int_{-d / 2}^{d / 2} \frac{1}{\sqrt{2 \pi \sigma^{2}}} e^{-\frac{x^{2}}{2 \sigma^{2}}} d x \int_{-d / 2}^{d / 2} \frac{1}{\sqrt{2 \pi \sigma^{2}}} e^{-\frac{y^{2}}{2 \sigma^{2}}} d y \\
& +\frac{1}{2} \int_{-d / 2}^{d / 2} \frac{1}{\sqrt{2 \pi \sigma^{2}}} e^{-\frac{x^{2}}{2 \sigma^{2}}} d x \int_{d / 2}^{3 d / 2} \frac{1}{\sqrt{2 \pi \sigma^{2}}} e^{-\frac{y^{2}}{2 \sigma^{2}}} d y \\
& =1-\frac{7}{2} Q\left(\frac{d}{2 \sigma}\right)-Q\left(\frac{3 d}{2 \sigma}\right)+\frac{3}{2} Q^{2}\left(\frac{d}{2 \sigma}\right)+Q\left(\frac{d}{2 \sigma}\right) Q\left(\frac{3 d}{2 \sigma}\right) .
\end{aligned}
$$

Case H: There is one symbol whose decision region is region $\mathrm{H}$, and the correct probability of each symbol is as follows:

$$
\begin{aligned}
P_{C H} & =\int_{-d / 2}^{\infty} \frac{1}{\sqrt{2 \pi \sigma^{2}}} e^{-\frac{y^{2}}{2 \sigma^{2}}} d y \int_{-d / 2}^{y-2 d} \frac{1}{\sqrt{2 \pi \sigma^{2}}} e^{-\frac{x^{2}}{2 \sigma^{2}}} d x \\
& \approx \int_{-d / 2}^{3 d / 2} \frac{1}{\sqrt{2 \pi \sigma^{2}}} e^{-\frac{y^{2}}{2 \sigma^{2}}} d y \int_{-d / 2}^{\infty} \frac{1}{\sqrt{2 \pi \sigma^{2}}} e^{-\frac{x^{2}}{2 \sigma^{2}}} d x \\
& +\frac{1}{2} \int_{3 d / 2}^{\infty} \frac{1}{\sqrt{2 \pi \sigma^{2}}} e^{-\frac{y^{2}}{2 \sigma^{2}}} d y \int_{-d / 2}^{\infty} \frac{1}{\sqrt{2 \pi \sigma^{2}}} e^{-\frac{x^{2}}{2 \sigma^{2}}} d x \\
& =1-2 Q\left(\frac{d}{2 \sigma}\right)-\frac{1}{2} Q\left(\frac{3 d}{2 \sigma}\right)+Q^{2}\left(\frac{d}{2 \sigma}\right)+\frac{1}{2} Q\left(\frac{d}{2 \sigma}\right) Q\left(\frac{3 d}{2 \sigma}\right) .
\end{aligned}
$$

Above all, the average correct probability of SCC can be calculated as

$$
\begin{aligned}
\bar{P}_{C}= & \frac{1}{L}\left\{2 P_{C A}+\frac{(\lambda-2)(\lambda-3)}{2} P_{C B}+2(\lambda-2) P_{C C}\right. \\
& \left.+P_{C D}+P_{C E}+\frac{\lambda-3}{2} P_{C F}+(\lambda-2) P_{C G}+P_{C H}\right\},
\end{aligned}
$$

where $\lambda=\sqrt{2 L}-1$, then the symbol error probability of SCC is

$$
\begin{aligned}
P_{e} & =1-\bar{P}_{C} \\
& =\frac{8 L-7 \sqrt{2 L}+2}{2 L} Q\left(\frac{d}{2 \sigma}\right)+\frac{\sqrt{2 L}-2}{L} Q\left(\frac{3 d}{2 \sigma}\right) \\
& -\frac{8 L-13 \sqrt{2 L}+10}{2 L} Q^{2}\left(\frac{d}{2 \sigma}\right)-\frac{3(\sqrt{2 L}-2)}{2 L} Q\left(\frac{d}{2 \sigma}\right) Q\left(\frac{3 d}{2 \sigma}\right) .
\end{aligned}
$$

This completes the proof of Theorem 3.

\section{REFERENCES}

[1] T. Komine and M. Nakagawa, "Fundamental analysis for visible-light communication system using LED lights," IEEE Trans. Consumer Electronics, vol. 50, pp. 100-107, Feb. 2004.

[2] A. Jovicic, J. Li, and Tom Richardson, "Visible light communication: opportunities, challenges and the path to market,"IEEE Commun. Magaz.,vol. 51, no. 12, pp. 26-32, Dec. 2013. 
[3] S. K. Routray, “The changing trends of optical communication,”IEEE Potentials, vol. 33, no. 1, pp. $28-33$, Jan. 2014.

[4] H. Burchardt, N. Serafimovski, D. Tsonev, S. Videv, and H. Haas,’VLC: Beyond Point-to-Point Communication,’IEEE Commun. Magaz.,vol. 52, no. 7, pp. 98-105, July 2014.

[5] L. Zeng, D. OBrien, H. Minh, G. Faulkner, K. Lee, D. Jung, Y. Oh, and E. T. Won, "High data rate multiple input multiple output (MIMO) optical wireless communications using white LED lighting," IEEE J. Sel. Areas Commun., vol. 27, no. 9, pp. 1654-1662, Dec. 2009.

[6] Y. J. Zhu, W. F. Liang, J. K. Zhang, and Y. Y. Zhang, "Space-collaborative constellation designs for MIMO indoor visible light communications," IEEE Photon. Technol. Lett., vol. 27, no. 15, pp. 1667-1670, Aug. 2015.

[7] H. B. Cai, J. Zhang, Y. J. Zhu, J. K. Zhang, and X. Yang, "Optimal Constellation Design for Indoor $2 \times 2$ MIMO Visible Light Communications,” IEEE Commun. Lett., vol. 20, no. 2, pp. 264-267, Feb. 2016.

[8] M. Safari and M. Uysal, "Do we really need OSTBCs for free-space optical communication with direct detection?" IEEE Trans. Wireless Commun., vol. 7, no. 11, pp. 4445-4448, Nov. 2008.

[9] J. M. Kahn and J. R. Barry, "Wireless infrared communications," Proc. IEEE, vol. 85, no. 2, pp. 265C-298, Feb. 1997.

[10] T. Fath and H. Haas, "Performance comparison of MIMO techniques for optical wireless communications in indoor environments," IEEE Trans. Commun., vol. 61, no. 2, pp. 733-742, Feb. 2013.

[11] R. Mesleh, H. Haas, S. Sinanovic, C. W. Ahn, and S. Yun, "Spatial modulation," IEEE Trans. Veh. Technol., vol. 57, no. 4, pp. 2228-2241, July 2008.

[12] W. O. Popoola, E. Poves, and H. Haas, "Spatial pulse position modulation for optical communications," J. Lightwave Technol., vol. 30, no. 18, pp. 2948-2954, Sep. 2012.

[13] T. Chen, L. Liu, B. Tu, Z. Zheng, and W. Hu,"High-spatial-diversity imaging receiver using fisheye lens for indoor MIMO VLCs," IEEE Photon. Technol. Lett., vol. 26, no. 22,pp. 2260-2263, Nov. 2014.

[14] A. Nuwanpriya, S.-W. Ho, and C. S. Chen, "Indoor MIMO visible light communications: novel angle diversity receivers for mobile users," IEEE J. Sel. Areas Commun., vol. 33, no. 9, pp. 1780-1792, Sep. 2015.

[15] A. A. Saed, S.-W. Ho and C. W. Sung, "Adaptive Modulation for Two Users in VLC," in Proc. IEEE Globecom Workshop, San Diego, USA, Dec. 2015.

[16] S. Hranilovic and F. R. Kschischang, "Optical intensity-modulated direct detection channels: Signal space and lattice codes," IEEE Trans. Inf. Theory, vol. 49, no. 6, pp. 1385-1399, Jun. 2003.

[17] M. Beko, and R. Dinis, "Systematic method for designing constellations for intensity-modulated optical systems," J. OPT. Commun. Netw.,vol. 6, no. 5, pp. 449-458, May 2014.

[18] J. Karout, E. Agrell, K. Szczerba, and M. Karlsson, "Optimizing constellations for single-subcarrier intensity-modulated optical systems," IEEE Trans. Inf. Theory, vol. 58, no. 7, pp. 4645-4659, July 2012. 